\title{
Pattern Separation in the Hippocampus: Distinct Circuits under Different Conditions
}

\begin{abstract}
Pattern separation is a fundamental hippocampal process thought to be critical for distinguishing similar episodic memories, and has long been recognized as a natural function of the dentate gyrus (DG) supporting autoassociative learning in CA3. Understanding how neural circuits within the DG-CA3 network mediate this process has received much interest, yet the exact mechanisms behind remain elusive. Here we argue for the case that sparse coding is necessary but not sufficient to ensure efficient separation and, alternatively, propose a possible interaction of distinct circuits which, nevertheless, act in synergy to produce a unitary function of pattern separation. The proposed circuits involve different functional granule-cell populations, a primary population mediates sparsification and provides recurrent excitation to the other populations which are related to additional pattern separation mechanisms with higher degrees of robustness against interference in CA3. A variety of top-down and bottom-up factors, such as motivation, emotion, and pattern similarity, controls the selection of circuitry depending on circumstances. According to this framework, a computational model is implemented and tested against model variants in a series of numerical simulations and biological experiments. The results demonstrate that the model combines fast learning, robust pattern separation and high storage capacity. It also accounts for the controversy around the involvement of the DG during memory recall, explains other puzzling findings, and makes predictions that can inform future investigations.
\end{abstract}

\section{KEY WORDS}

computational model; dentate gyrus; episodic memory; hippocampus; pattern separation

\section{INTRODUCTION}

Much of what we actually remember is related to unique, personally experienced events, which are known as episodic memories (Tulving, 1972). Despite a high degree of similarity between episodic memories, e.g. common, familiar people, places or similar routines, we are usually able to remember distinctive characteristics of closely related but distinct episodes. This ability critically depends on the hippocampus (Scoville and Milner, 1957; Eichenbaum, 2004; Treves et al., 2008; Rolls, 2013), and is thought to be mediated by a process of pattern separation, by which afferent inputs from the entorhinal cortex (EC) are transformed into distinct, relatively non-overlapping representations (Yassa and Stark, 2011). Early studies of hippocampal CA3-like recurrent networks have clearly demonstrated the importance of sparsely encoded input patterns for increasing storage capacity and for preventing interference from similar memories (Amari, 1989; Knoblauch et al., 2010). It has long been conjectured, therefore, that hippocampal pattern separation might originate upstream from CA3, more precisely, in the DG (McNaughton and Nadel, 1990; Treves and Rolls, 1994; Treves et al., 2008). Solid research over the ensuing years has substantiated the putative role of the DG in pattern separation, with evidence from electrophysiological, lesion and immediate-early-gene imaging studies in rodents (Gilbert et al., 2001; Lee and Kesner, 2004; McHugh et al., 2007; Leutgeb et al., 2007; Hunsaker et al., 2008; Goodrich-Hunsaker et al., 2008) and from functional magnetic resonance imaging studies in humans (Bakker et al., 2008; Berron et al., 2016). The candidate mechanisms of how pattern separation is performed in the DG and how the effect of separation is maintained downstream in CA3, however, remain a matter of debate.

\section{Sparsification}

The granule cells of the DG have unusually low firing rates and largely outnumber both their presynaptic EC projection cells and their postsynaptic CA3 pyramidal cells (In each rat hemisphere, EC projections from 110,000 EC layer II cells diverge onto 1.2 million granule cells whose axons converges on 250,000 CA3 pyramidal cells (West et al., 1991; Witter, 2010), with only 1-2\% of granule cells exhibiting activity (Chawla et al., 2005)). Based on these unique properties, granule cells are suggested to sparsely recode EC inputs such that only a minority of the granule cell population can be active, allowing for their neural representations in the DG to be orthogonalized or at least almost orthogonalized (Chawla et al., 2005; Leutgeb et al., 2007; Deng et al., 2013). The direct link between sparseness and pattern separation was originally described by David Marr in his earlier studies of the cerebellum (Marr, 1969), and subsequently inspired hippocampal theories (Marr, 1971; McNaughton and Nadel, 1990; O’Reilly and McClelland, 1994). 
Most Marr-like models assume, either explicitly or implicitly, an inhibitory control over the activity of granule cells through activation of local interneurons (Treves and Rolls, 1994; Myers and Scharfman, 2009; Aimone et al., 2009). The central idea is that EC inputs excite granule cells which, in turn, excite interneurons that inhibit other granule cells, making the most excited granule cells compete with the less excited ones. The sparseness of DG neural codes is inversely related to the proportion of granule cells that survive inhibition (O’Reilly and McClelland, 1994). Some authors have further suggested that the plasticity observed at the perforant path-granule cell synapses (Bliss and Lomo, 1973) could help with this type of recoding through statistical learning (Treves and Rolls, 1994; Rolls, 2013). Such plasticity-mediated computations have largely been developed in competitive learning theories (Rolls and Treves, 1998) and proved very efficient in a wide range of incremental learning paradigms (O’Reilly and Rudy, 2001; Gluck et al., 2003).

In contrast, episodic learning challenges classical competitive learning theories because episodes occur only once and, therefore, cannot be learned through interleaved repetitions; their learning should be fast, often even in only single trial (Morris, 2001; Moser and Moser, 2003). This is a hard learning problem which has long constrained episodic memory models to relatively simple mechanisms of sparsification (McNaughton and Nadel, 1990; Weisz and Argibay, 2009; Myers and Scharfman, 2009, 2011). Such fast "one-shot" mechanisms could decrease, but not necessarily eliminate, input similarity; extant overlap among output representations is most likely to increase as a function of input similarity and number of stored representations (Weisz and Argibay, 2009; Myers and Scharfman, 2011). This does not necessarily discount these mechanisms, but might suggest that a rapid process of sparsification is necessary but not sufficient to ensure efficient storage and recall. If this is the case, then the intriguing question is, "what additional computations does the DG execute to ensure efficient separation even in one single trial?"

\section{Other Potential Mechanisms}

Processing routes within the DG are complex and its glutamatergic cell populations are heterogeneous, at least, in two basic aspects (Fig. 1). First, new granule cells are continuously generated throughout adulthood, and as they mature, newborn cells display unique physiological properties which distinguish them from older adult-born cells and cells born early during development (Ge et al., 2007; Dieni et al., 2013). Second, mossy cells, which are located exclusively in the hilus under the granule cell layer, form a distinct glutamatergic cell population in the DG, although with much less abundance than granule cells ( 50,000 mossy cells in one hemisphere of the rat hippocampus (West et al., 1991)). Like granule cells, mossy cells rarely show mutual connections, but they form rich connectivity with other cell types in the DG (Acsàdy et al., 1998; Buckmaster et al., 1996; Larimer and Strowbridge, 2008). In particular, granule cell axons, the mossy fibers, innervate mossy cells and GABAergic neurons, before reaching CA3. In turn, mossy cells primarily innervate distant ipsilateral and contralateral granule cells, but they also synapse onto different types of local interneurons that inhibit granule cells (Scharfman, 2016). This pattern of connectivity creates multiple positive and negative feedback loops in the DG, which makes it difficult to reveal the ultimate effect of mossy cells on the activation of granule cells (Scharfman, 2016). The complexity of addressing this issue is further increased by the fact that hilar cells receive a feedback projection from CA3 (Ishizuka et al., 1990; Li et al., 1994; Scharfman, 2007), and different neuromodulatory inputs (Amaral et al., 2007), which may bias their engagement under different conditions.

Plastic changes have been reported in both granule cell-mossy cell and mossy cell-granule cell synapses (Lysetskiy et al., 2005; Hetherington et al., 1994). This plasticity along with the pattern of connectivity described above provide initial support for the granule cell-mossy cell-granule cell circuit as a potential substrate for a second level of information processing in the DG. In line with this view, a number of studies have explored the functional significance of hilar circuitry (Buckmaster and Schwartzkroin, 1994; Buckmaster et al., 1996; Lisman, 1999) of which some are suggestive of a role in pattern separation (Myers and Scharfman, 2009; Aimone et al., 2009; Jinde et al., 2012; Senzai and Buzsáki, 2017). In a very interesting model, for instance, Myers and Scharfman (2009) proposed that hilar cells might bias competition between granule cells, providing a mechanism to regulate pattern separation in the DG. The model exhibited efficient pattern separation in the DG, but when extended later to include a CA3 network that is small relative to the DG, the model showed poor pattern separation evidenced by very low storage capacity in CA3 (Myers and Scharfman, 2011).

Influential theoretical models posit that pattern separation may be facilitated in CA3 by the strong and relatively focused aspects of mossy fibers which relay sparse patterns of activity from the DG (O'Reilly and McClelland, 1994; Treves and Rolls, 1994). These characteristics are presumed to enable efficient separation of CA3 patterns without the need for plastic changes at mossy fiber-CA3 synapses. Such a solution would be especially valuable for episodic learning, but other elements appear to be missing from this picture. Although the convergent nature of mossy fibers potentially reduces the 
effect of separation achieved in the DG (Myers and Scharfman, 2011), they appear to elicit at the same time strong recruitment of CA3 interneurons (Acsàdy et al., 1998), which may be key players in CA3 pattern separation (Ruediger et al., 2011). In their model, Myers and Scharfman (2011) also showed that the activation of inhibitory feedback connections from CA3 to DG granule cells improves both pattern separation and storage capacity in CA3. Nonetheless, empirical data are lacking in the precise nature of CA3 backprojection and its potential contribution to pattern separation.

Relatively much more attention has been paid to the possibility that adult-born granule cells might support pattern separation under conditions of presumably high level of interference (Clelland et al., 2009; Nakashiba et al., 2012). Immature granule cells, which exhibit a transient period of increased excitability and synaptic plasticity (Ge et al., 2007), are widely believed to function differently from their mature counterparts. A potent idea is that immature neurons may be preferentially recruited to encode new events, and when becoming mature, they preferentially respond to those events that they experienced during their development (Wiskott et al., 2006; Kee et al., 2007; Aimone et al., 2009); other studies take this idea to the extreme that mature granule cells are functionally retired (Alme et al., 2010). This view contrasts with subsequent experiments suggesting that young and mature granule cells are equally likely to be recruited in memory processes, see (Lopez-Rojas and Kreutz, 2016) for a review.

\section{A Synergistic View of Pattern Separation}

What is clear from the above considerations is that DG intrinsic neurocircuits might underlie additional mechanisms which go beyond simple sparsification through the most abundant granule cells. In actual fact, granule cells themselves are not homogeneous and their activity is controlled by recurrent excitatory and inhibitory circuits which might have profound effects on the functional responsiveness of these cells. While attempts have been made to construct models that include all or most of these aspects, DG pattern separation is discussed in these models as a global function supported by a single, even if sophisticated, circuitry. Instead, we develop in the present work the idea that hippocampal pattern separation involves distinct, but highly intertwined, circuits underlying different mechanisms of decorrelation with different degrees of robustness against interference. In our framework, new patterns are either made sparser or completely orthogonalized depending on specific factors, such as pattern similarity, emotion and mood states. Sparsification is rapidly achieved through a large population of granule cells with strong excitatory drive onto CA3 pyramidal cells. In some cases, convergent inputs from the DG to CA3 are accompanied by feedforward inhibition in order to counteract potential interference in CA3. In other extreme cases, a functionally distinct population of granule cells forms a second-level representation of a larger population of granule cells and provides the basis for a complete orthogonalization in CA3. Here we present a unifying computational framework which shows how known details of the DG-CA3 circuitry might support these pattern separation mechanisms and demonstrates their relevance for rapid yet accurate encoding and retrieval of information from one-time experiences.

\section{A COMPUTATIONAL MODEL OF THE DG-CA3 NETWORK}

\section{Generalities}

The purpose of the model is to provide a computational framework for identifying key pathways in the DG-CA3 circuitry that are likely to be involved in its putative function of pattern separation, and for explaining how the neural responses differ under specific conditions. The architecture of the present model is derived from known anatomical and physiological characteristics of the intrinsic circuitry of the DG and its connectivity with CA3 (Fig. 1 and 2). In particular, we demonstrate through the model how a simple "sparsification" process through the most abundant granule cells would act in concert with additional circuits in the DG-CA3 network to further boost the effect of pattern separation in CA3. We also explore through the model a potential role for the hilus as a locus where signals from extra- and intrahippocampal structures are gathered together to harness these different circuits, allowing for different pattern separation mechanisms to be recruited under different conditions. In actual cases, a variety of top-down and bottom-up factors may affect the level of load on pattern separation; input similarity (how similar an input pattern is to already established memories) is among the most prominent and highly studied (e.g., Gilbert et al. (2001); Clelland et al. (2009)). Here we mostly focus on this factor to exemplify the general principle of the model, other factors will be discussed further later in the relevant sections.

We remark that the basic architecture of the present model incorporates previous key assumptions regarding the functional principles of the DG-CA3 circuitry (e.g., Marr (1971); McNaughton and Nadel (1990); O’Reilly and McClelland (1994); Treves and Rolls (1994); Hasselmo et al. (1996); Rolls (2013)), including notably that: 1) The CA3 network acts as an autoassociative memory with dense recurrent collaterals which is capable of storing and subsequently retrieving memory 
traces. 2) The DG creates a sparse coding of EC inputs and drives CA3 neural activity during storage to construct new attractors representing novel inputs. 3) The direct EC-CA3 perforant pathway provides CA3 with retrieval cues, while the indirect disynaptic input from EC to CA3 via the DG is not directly involved during recall. 4) Novelty of EC inputs induces circuit dynamics that favor learning over recall, through cholinergic and GABAergic projections from the basal forebrain. These basic assumptions lead to a standard model which mirrors fundamental properties of the anatomical and functional correspondences of the DG-CA3 circuitry. Our model means to advance this view by considering different populations of granule cells with different patterns of connectivity with CA3 cells. The model is described next with an emphasis on the specific aspects that diverge from single-population models and the ways in which they adjust the functional and dynamical assumptions stated above. Additional implementation details are provided in the appendix.

\section{Network Structure and Connectivity}

The architecture of the model is consistent with the known anatomy of the hippocampus (Fig. 1), and is shown schematically in Fig. 2. The model includes networks for the DG and CA3 with both receiving common inputs from the EC. A core assumption of the model is that DG granule cells form functionally heterogeneous neuronal populations that differ in their effects on CA3 pyramidal cells. From this perspective, the model defines two granule-cell populations in the DG network: a large population of granule cells, PPGC (Perforant Path-driven Granule Cells), which are driven by inputs from the EC, and a smaller population of granule cells, HGC (Hilar-driven Granule Cells), which are solely driven by their inputs from hilar mossy cells, not by EC inputs. This assumption can be supported by the fact that the terminal zones of these two afferent inputs are spatially segregated on the dendrites of granule cells (Amaral et al., 2007; Witter, 2010). The perforant path fibers from the EC terminate in the outer two-thirds of the molecular layer of the DG, while the axons of mossy cells terminate in the inner third of the molecular layer. Also a subtype of GABAergic neurons in the DG (MOPP and HIPP) have axons restricted to the terminal zone of the perforant path (Amaral et al., 2007; Freund and Buzsáki, 1996). Therefore, it seems reasonable to assume that a population of granule cells may be subject to a relatively strong dendritic inhibition coaligned with EC inputs, preventing dendritic integration of EC excitation and rendering this population exclusively responsive to excitation from mossy cells. It also seems likely that such specificity may arise via an activity-dependent learning process during maturation of newborn neurons in the DG. We elaborate on these and other possibilities later in the discussion.

The model assumes 200 cells in the EC, 1000 PPGC and 120 HGC in the DG, and 120 MC (mossy cells) in the hilus of the DG. The cell numbers are scaled down by approximately 1000 the size of the rat hippocampus (West et al., 1991; Witter, 2010); a scale that is usually used in hippocampal models, e.g. (Myers and Scharfman, 2011), to maintain computational tractability while ensuring the significance of simulation results. The EC-DG connectivity is sparse in the substrate; it is estimated that each granule cell receives from a random 2-3\% of the EC cells, and that $10 \%$ of these inputs need to be active to discharge the cell (McNaughton91, Witter, 2010). Because our relatively small-scale model does not allow to strictly adhere to these estimates, we set the density of EC-DG projection to 25\%, meaning that each PPGC/HGC receives input from a random determined $25 \%$ of the EC cells. The level of tonic inhibition has been fixed such that a PPGC can only fire in response to activation of at least $10 \%$ of its EC afferents. Plasticity at the EC-GC synapses is not considered in the present model; all synapses have static random weights but the influence of EC inputs onto HGC is suppressed by a local interneuron which receives direct input from all EC cells. The model organizes both PPGC and HGC into a series of 10 equally-sized non-overlapping clusters, each containing 100 PPGC and 12 HGC; such clusters, as proposed in (Myers and Scharfman, 2009), can be regarded as an emerging aspect of the lamellar organization of mossy fiber-CA3 projections which run transversely into a series of very narrow septotemporal segments of the hippocampus (Andersen et al., 1971; Amaral et al., 2007). Sparsification is implemented through a simple version of a lateral inhibitory winner-take-all (WTA) competition in which all but the most strongly-activated PPGC within each cluster are silenced. PPGC have a fully connected projection to MC and this projection is endowed with Hebbian plasticity. MC compete in a WTA fashion that allows at most one MC to be activated at any one time, and project onto HGC in one-to-one relationships. This is a simplification of the one-to-many projection of mossy cells that excite granule cells (Buckmaster et al., 1996), but does not impact the functional principle of the model; a HGC can be thought of as a unit representing a small number of granule cells. The synaptic weights between MC and HGC are constant and set so that a sufficiently excited MC can automatically evoke a postsynaptic response in HGC. Such a potent excitation from mossy cells onto granule cells has been recorded in in vitro slices, especially under conditions of disinhibition (Scharfman, 1995; Jackson and Scharfman, 1996). It has also been reported that plasticity of the dentate recurrent excitation network facilitates mossy cell excitatory actions in vivo (Kleschevnikov and Routtenberg, 2003). 
In CA3, the model contains large and small populations of CA3 pyramidal cells, PPCA3 and HCA3, respectively. This distinction is based on anatomical and neurophysiological findings that CA3 can be divided into subregions located at different transverse positions, CA3a, b, and c, with apparent differences in their connectivity (Ishizuka et al., 1990; Li et al., 1994; Scharfman, 2007). No data exist regarding the relative number of cells in these CA3 regions but, in the model, as will become clear shortly, the number of HCA3 is limited by the relatively small number of mossy cells, and set to 50 by default, while the number of PPCA3 has been set to 300. Like DG granule cells, both PPCA3 and HCA3 are each equally divided into 10 clusters, each containing 30 PPCA3 and 5 HCA3. Only PPCA3 receive projections from PPGC through sparse, lamellar and nonplastic connections; each PPGC in a given cluster targets a PPCA3 within the corresponding cluster in CA3 and, on average, each PPCA3 is linked to three or four PPGC. The direct EC input only goes to PPCA3; each PPCA3 receives from a random $25 \%$ of the EC cells. PPCA3 are interconnected by recurrent connections in a nonlamellar fashion; each PPCA3 receives from a random 50\% PPCA3 sampled from the entire population. The density of this projection has been chosen twice higher than the density of EC-PPCA3 afferents to accommodate the fact that collateral synapses provide the largest number of synapses on the dendrites of CA3 pyramidal cells (Witter, 2010). It has also been reported that such a high density of recurrent collaterals can be estimated in CA3a but gradually decreases to near zero in CA3c (de Almeida et al., 2007). Both EC-PPCA3 and PPCA3 recurrent projections are associatively modifiable by Hebbian learning.

The backprojection from PPCA3 to MC integrates the global level of activation in PPCA3 and triggers the recruitment of $\mathrm{MC}$ under specific conditions. We hypothesize that $\mathrm{MC}$ are divided into two subpopulations, $\mathrm{MC}_{1}$ and $\mathrm{MC}_{\mathrm{h}}$, with low- and high-activation thresholds, $\theta_{1}$ and $\theta_{h}$, in response to PPCA3 cells. Both $M C_{l}$ and $\mathrm{MC}_{\mathrm{h}}$ behave as a WTA network and $\mathrm{MC}_{\mathrm{h}}$ exert an inhibitory control masking $\mathrm{MC}_{1}$ activity when they fire simultaneously. The only available evidence in support of such a dichotomy in mossy cell responsiveness comes from empirical studies showing that a subset of mossy cells have a low threshold to perforant path stimulation in hippocampal slices, and predicting that this subset may correspond to the small subset of mossy cells that appear to be spontaneously active in vivo (Scharfman, 1991, Duffy et al., 2013). Based on these data, it stands to reason that such behavior might result from differential innervation of local hilar interneurons by CA3 backprojection, making one of two subpopulations of mossy cells $\left(\mathrm{MC}_{1}\right)$ more easily activated by CA3 than is the other subpopulation $\left(\mathrm{MC}_{\mathrm{h}}\right)$. Obviously, further testing needs to be done to confront our hypothesis (i.e., to determine which inputs could facilitate the activity of mossy cells and whether CA3 inputs could trigger a similar effect on mossy cells).

In the model, the two groups of mossy cells, $\mathrm{MC}_{1}$ and $\mathrm{MC}_{\mathrm{h}}$, do not differ in their connectivity to HGC, but HGC themselves do differ with respect to their connectivity to CA3. HGC that are driven by $\mathrm{MC}_{1}$ exert a feedforward inhibition on PPCA3 via Hebbian modifiable inhibitory synapses; this inhibition occurs on the distal dendrites of PPCA3 and thereby has scant or no effect on their activation by mossy fiber input. HGC that are driven by $\mathrm{MC}_{\mathrm{h}}$ make one-to-one excitatory synapses on HCA3 with constant synaptic weights (for this reason the number of $\mathrm{MC}_{\mathrm{h}}$ is set equal to the number of $\mathrm{HCA3}$, i.e. 50), and HCA3, in turn, mediate a global inhibition on all PPCA3. This connectivity is obviously a simplification of mossy fiber projections to both CA3 pyramidal cells and interneurons (Acsàdy et al., 1998), meant to more clearly delineate the specific functional contributions of HGC. For instance, the global inhibition from HCA3 onto PPCA3 can be replaced or fostered by direct inhibition from HGC that are driven by $\mathrm{MC}_{\mathrm{h}}$ onto PPCA3 without affecting the model's fundamentals.

\section{Functional Dynamics}

The model has two modes of operation: a "storage mode" which is prioritized when inputs from the EC are novel, and a "recall mode" in which the model settles otherwise. Support for these functional modes derives from an elegant series of studies by Hasselmo and colleagues (see, e.g., Hasselmo et al. (1996)) linking cholinergic and GABAergic modulatory effects to circuit dynamics in the hippocampus. Increases in hippocampal acetylcholine release, which are correlated with novelty detection (Aloisi et al., 1997), have been shown to suppress transmission at intrinsic synapses more than transmission at extrinsic inputs from the EC; specifically, suppressing CA3 recurrent inputs would be relevant to the storage of novel inputs without interference from previously stored memories. This idea has been further elaborated by the proposal of hippocampal theta rhythm, which is modulated by cholinergic and GABAergic inputs from the medial septum, as a mechanism for rapid transitions between encoding and recall dynamics (Hasselmo et al., 2002).

It is widely held that the hippocampus generates its own novelty signal and uses it to incite learning dynamics through the septohippocampal loop (Hasselmo et al., 1996). It is difficult, as yet, to decode with certainty the locus of novelty detection in the hippocampus; both CA3 and CA1 are compelling candidates (Lee, 2005; Vago and Kesner, 2008): a weak recall in CA3 may reflect novelty, but also CA1 may serve as a basis for mismatch comparison of converging inputs from the EC and CA3. With both computations the hippocampus would be able to learn new inputs that differ from previously stored 
memories, but would be less obviously able to deal with situations where familiar, but unexpected inputs need to be learned as separate from similar past memories (e.g., as needed for the distinction between similar contexts in fear conditioning paradigms (Nakashiba et al., 2012)).

Within our framework, we consider that the shift from recall to learning dynamics is not solely determined by the similarity between incoming and existing information, but also by other signals from extra-hippocampal structures attributing behaviorally significant values to environmental states, generally considered as emotional, motivational, perceptual or attentional signals. The current instance of the model incorporates this consideration in simple ways: a switch to storage mode is forced either by an external signal regardless of input similarity or by a matching process that depends on an externally-controlled threshold, $\mathrm{v}_{\mathrm{m}}$, for deciding whether a match or a mismatch should be considered between CA3 recall activity and previously stored memory traces.

Instead of using recall activity in CA3 as a signal to shift between learning and recall modes, our model uses it - along with other emotional/motivational modulatory signals - at the early stage of learning to drive circuit dynamics during the storage of novel inputs. Specifically, CA3 activity is taken as a quantitative indicator of the potential of the newly to-be-stored information to interfere with previously stored memories; the potential risk of interference is formulated as a function of the summed activity of the whole population of PPCA3, $\Sigma$. Depending on the strength of this signal $(\Sigma)$, three scenarios are possible in the model:

Sparsification If no, or weak, activity is elicited in PPCA3 during recall $\left(\Sigma \leq \theta_{1}\right)$, MC remain silent and encoding occurs in the sparsification circuit. Input from the EC provides excitatory drive to PPGC which produce by their competitive interactions sparse outputs and via their mossy fibers force a new representation on PPCA3 to be stored in the network; storage takes place through Hebbian associative plasticity at EC-PPCA3 and PPCA3-PPCA3 synapses. During encoding, synaptic transmission is suppressed at both the perforant path and recurrent inputs to PPCA3 to avoid interference from retrieval of existing memories. It is possible, however, to obviate the need for this suppression at the modifiable synapses as long as the threshold, $\theta_{1}$, is low enough to ensure interference-free learning in PPCA3. During recall, synaptic transmission is selectively suppressed at PPGC mossy fiber synapses allowing the other two inputs to dominate the drive of PPCA3 and to support immediate retrieval of the stored patterns.

Inhibition A limited scope for interference can be detected if the level of activity elicited in PPCA3 by an EC input during recall is higher than normal but not high enough for signaling a need for absolute orthogonalization $\left(\theta_{1}<\Sigma \leq \theta_{h}\right)$. In this case learning has two phases. In the first phase, the network maintains recall dynamics and the feedback signal from CA3 activates a $M C_{1}$ in the hilus. This activation causes Hebbian learning to occur at PPGC-MC heteroassociative synapses, and concomitantly, the activated $\mathrm{MC}_{1}$ undergoes a state of saturation to ensure that the same mossy cell will not be recruited more than once by CA3. In the second phase, the network makes a complete switch to learning dynamics and PPGC inputs through mossy fibers to PPCA3 and to MC dominate the activity in the network. Both EC-PPCA3 and PPCA3-PPCA3 synapses undergo Hebbian plasticity to store the representation set up by PPGC mossy fibers in PPCA3. At the same time, PPGC provide $\mathrm{MC}_{1}$ that responded to CA3 backprojection during the first phase with a strong drive leading to the activation of the corresponding HGC and its inhibitory action on PPCA3. The co-occurrence of postsynaptic firing in PPCA3 triggers synaptic depression at the inhibitory synapses made onto active PPCA3. This implies that only silent PPCA3 cells remain under the inhibitory control imposed by HGC on PPCA3, ensuring that the activity elicited in PPCA3 will be suppressed during the retrieval of the stored memory.

It is now evident that mossy fibers have unique morphological and physiological properties accounting for differential control of synaptic plasticity and transmission along the same axon, depending on its postsynaptic targets (Acsády et al., 1998; McBain, 2008). Little is known, however, about the functional implications of these specialized terminals for memory encoding and retrieval. In our model, we assume that mossy fibers that target MC and CA3 interneurons are active during recall while PPGC mossy fibers onto PPCA3 are selectively suppressed. This enables the DG to work in tandem with CA3, with a net inhibitory action on PPCA3, leading to reasonably accurate recall of the neural representation of a memory that has been encoded throughout the inhibition circuit.

Orthogonalization When the feedback signal from CA3 to MC is turned up very high ( $\left.\Sigma>\theta_{\mathrm{h}}\right)$, it is primarily because a high degree of similarity exists between the current input and, at least, one of the previously stored inputs. Storing the current input in the PPCA3 network - even if represented by a strictly separate subpopulation of PPCA3 cells - is most likely to retroactively interfere with the retrieval of those other memories due to the associative plasticity at EC-PPCA3 
synapses. There should be, then, some additional mechanism(s) to keep earlier learning from being disturbed by storing of new inputs.

In the present model, we suggest that the hippocampus might invoke additional pathways to encode and retrieve EC inputs that present a high potential for interference. The network dynamics are quite similar to what we described above for the inhibition circuit. When CA3 sends back a sufficiently strong signal to the hilus, a $\mathrm{MC}_{\mathrm{h}}$ responds to this signal and learning takes place at PPGC-MC synapses. Synaptic transmission at mossy fiber synapses is then promoted and causes $\mathrm{MC}_{\mathrm{h}}$ to become more active under intensive excitatory drive from PPGC. The corresponding HGC and HCA3 become activated in turn, while all PPCA3 fall silent under the powerful inhibitory action of HCA3 on PPCA3 during learning (and also during recall).

This circuit leads to a complete orthogonal representation for the current input which is spatially separate from other similar memories stored in the PPCA3 network. The direct perforant path is not responsible for the retrieval of the orthogonalized representation; instead, the indirect perforant input via the DG becomes directly involved in recall. Unlike PPGC mossy fibers onto PPCA3, synaptic transmission through mossy fiber inputs to HCA3 is enabled during both learning and recall. The argument stated above regarding the target-cell-dependent transmission at mossy fibre-CA3 synapses would still apply here based on the anatomical and neurophysiological heterogeneity of CA3 subregions (CA3a, b, and c).

\section{TESTING THE MODEL}

A series of numerical experiments were conducted to examine quantitative issues that are essential to refine the theoretical perspective of the model. The ability of the model to perform pattern separation has been analyzed assuming varying sized sets of input patterns with different degrees of correlation. The inputs were modeled as vectors of 200 EC cells with $10 \%$ activity (20 cells out of 200 are active (1) and the rest are silent (0)). The two working modes of learning and recall were separated by an external signal to ensure storage of all input patterns regardless of their similarity with each other. The model was trained to store, in a single pass, input patterns and, subsequently, tested for its ability to retrieve these patterns. We recorded the patterns of activity of DG and CA3 cells to quantify their individual contributions during storage and recall phases. Pattern separation was assessed by the extent to which the average similarity among the DG/CA3 output patterns is less than the average similarity among the EC input patterns. The quality of retrieval, which can be regarded as an indirect measure of the quality of pattern separation, was evaluated by computing the average similarity between the stored and recalled patterns. The normalized dot product, which calculates the cosine of the angle between two vectors, was used as a measure of the similarity between two activity patterns. This means that the more similar the two patterns, the closer the normalized dot product is to 1 ; whereas, in contrast, the less similar they are, the closer the normalized dot product is to 0 .

We compared the model, hereafter referred to as the S-I-O model, with three reduced alternatives. In the first, the basic model (S), only the basic circuitry of sparsification is included, similar to standard models of the hippocampus. The second and the third are models with either the neural circuitry of inhibition (S-I) or orthogonalization (S-O) included. All experiments were performed with the dual thresholding function being implemented in the model. The network parameters were set at default, except when investigating the relative contributions of the different circuits by varying MC firing thresholds. Exploring the effects of different MC firing thresholds was intended to simulate the modulatory influence that emotional/motivational states might exert on memory discrimination. For each experiment, 10 simulations were run with random initializations of the network connections. The results were averaged across simulations and reported as mean \pm standard error.

\section{Pattern Separation}

The first aspect we looked at was the effect of similarity between EC inputs on the degree of separation provided by the model. For this purpose, different pairs of EC input patterns were generated and the amount of overlap between each pair was manipulated by varying the percentage of active cells they share. Specifically, for each pair, a first pattern was randomly generated with 20 out of 200 cells being active, then a second pattern was constructed from the first one, by switching the state of fixed number (n) of randomly chosen cells that were active (1) to silent (0) and the state of the same number of randomly chosen cells that were silent to active. This means that the similarity between two input patterns will decrease for increasing values of $n$ (for $n=1,19$ of the 20 active EC cells will be active in both patterns, and then the input similarity measured by the normalized dot product is 0.95 ; while for $n=19$, only one of the 20 active EC cells are active in

both patterns, and the input similarity is 0.05). For each pair, the similarities between DG/CA3 responses were compared 
with the similarity between the corresponding input patterns (Fig. 3). Pattern separation occurs if the similarity measured at DG/CA3 outputs is less than the similarity measured at the input.

Fig. 3A shows that, when the similarity between the input patterns was low, both the S and S-I-O models demonstrated similar pattern separation capabilities. However, in the S model, pattern separation was weak in the DG, and completely failed in CA3, as input similarity was increased; in contrast, this increase in similarity did not appear to degrade pattern separation in the S-I-O model. To verify these impressions, an overall three-way ANOVA was conducted with model (S vs. S-I-O) and region (DG vs. CA3) as the between factors and input similarity as the within factor. The analysis yielded a main effect of model $\left(\mathrm{F}_{1,540}=482.76, \mathrm{P}<0.001\right)$, a main effect of region $\left(\mathrm{F}_{1,540}=164.14, \mathrm{P}<0.001\right)$, and a main effect of similarity $\left(\mathrm{F}_{19,180}=38.86, \mathrm{P}<0.001\right)$, with significant interaction effects (for all combinations, $\mathrm{P}<0.001$ ). Post-hoc analyses were subsequently performed to get more insight into these results.

Repeated-measures ANOVA confirmed that the S model showed both a significant decrease in pattern separation as input similarity increased $\left(\mathrm{F}_{19,180}=55.98, \mathrm{P}<0.001\right)$, and a significant difference between the DG and CA3 regions $\left(\mathrm{F}_{1,180}=307.20\right.$, $\mathrm{P}<0.001)$; there was also a significant similarity $\times$ region interaction $\left(\mathrm{F}_{19,180}=16.76, \mathrm{P}<0.001\right)$; this effect was only significant for inputs with high similarities (Input similarity $>0.6$, all $\mathrm{P}<0.01$ ) but not for lower similarities (all $\mathrm{P}>0.05$ ). In large part similar results were reported by earlier modeling studies, e.g., (O’Reilly and McClelland, 1994), which have suggested that CA3 pattern separation is not as robust as in the DG due to higher activity levels in CA3. While this suggestion may partly explain our results as well, we found that Hebbian plasticity in the direct EC-CA3 pathway accounts more for the degraded CA3 pattern separation in the basic model; during recall, much overlap between EC inputs caused significant interference in CA3 responses.

The S-I-O model checks for this effect ahead of time and allows for more elaborated processing in cases where a potential interference is identified. Specifically, pattern separation was performed strongly and quasi-equally for all pairs of inputs, regardless of their mutual similarity (One-way ANOVA, DG: $\mathrm{F}_{19,180}=5.56, \mathrm{P}<0.01$; $\mathrm{CA3}$ : $\mathrm{F}_{19,180}=1.58, \mathrm{P}=0.063$ ). Repeatedmeasures ANOVA suggested a significant similarity $\times$ region interaction $\left(\mathrm{F}_{19,180}=1.89, \mathrm{P}=0.017\right)$, but post-hoc tests revealed no significant difference between the DG and CA3 at most levels of input similarity. The significant effect emerged mainly from the highest level of similarity, due to the absolute orthogonalization in CA3 which gives rise to an output similarity of zero. The results also demonstrated a highly significant improvement in pattern separation abilities over the basic model in both regions. Post-hoc tests showed that the significant effect was mainly driven by pairs with high similarity (Input similarity $>0.6$, all $\mathrm{P}<0.05$ in the DG and CA3).

In Fig. 3B, it is possible to identify which of the three circuits in the S-I-O model has intervened to encode the second pattern in each pair of inputs. As expected, sparsification predominated at low input similarity, inhibition predominantly occurred at medium similarity, while orthogonalization predominated at high levels of similarity. The individual contributions of these circuits are evaluated separately using the S-I and S-O models (Fig. 3C and 3D). The effect of orthogonalization can clearly be seen at high levels of similarity (ANOVA with post-hoc tests, DG: all $\mathrm{P}<0.001$ for input similarity greater than 0.75 ; CA3: $\mathrm{P}<0.05$ for input similarity greater than 0.7 ). The effect of inhibition was not significant $(\mathrm{P}>.05)$. These results are not surprising and can be attributed to two factors. First, the default values of MC thresholds $\left(\theta_{1}\right.$ and $\theta_{\mathrm{h}}$ ) were selected such that inhibition should be activated at low levels of interference. Second, as a pattern-separation mechanism, inhibition is less robust than orthogonalization. Inhibition can protect the later pattern against interference, though at the cost of making the early "similar" pattern more vulnerable to interference. With orthogonalization, the early pattern is not affected by the orthogonalized pattern because both learning and retrieval of these two patterns involve separate pathways.

In order to clarify the latter point, we repeated the simulations outlined above with only one of the inhibition or orthogonalization circuits allowed to be activated at any level of interference. One way to achieve this is by changing the default values for MC thresholds; for example, the inhibition circuit can be completely disabled by setting $\theta_{1}=\theta_{\mathrm{h}}=0$, while the orthogonalization circuit can be completely disabled by setting $\theta_{\mathrm{l}}=0$ and $\theta_{\mathrm{h}}=1$. Fig. 4 depicts the results for these two extreme cases. If we contrast these results with Fig. 3A, we can see that inhibition (S-I) had a stronger tendency toward pattern separation than was the case with the basic model (S) (Paired tests, DG: all $\mathrm{P}<0.05$ for input similarity greater than 0.6; CA3: $\mathrm{P}<0.05$ for input similarity greater than 0.7 with one exception). On the other hand, inhibition exhibited significantly higher similarity between CA3 outputs than orthogonalization did (Paired tests, all $\mathrm{P}<0.05$ for input similarity greater than 0.55; Fig. 4). This is again because the retrieval of the early pattern was abolished by the concurrent storage of the later pattern on the direct EC-CA3 synapses. Recall led to a superimposition of the two stored memories, an effect that 
was masked by inhibition in the case of the later pattern, not the early one. Because orthogonalization does not alter learning at the direct EC-CA3 pathway, it does not affect retrieval of other non-orthogonalized patterns.

This pattern of results led us to question what may happen if the paired patterns are presented twice to the S-I model for learning purpose. Would the inhibition-mediated mechanism be able to reduce the retroactive interference caused by the storage of the second pattern in a pair? In order to address this question, we repeated the above simulation with two learning trials. On the second trial, learning was allowed to occur if the similarity between the retrieved pattern and the previouslystored pattern in CA3 was less than a matching threshold $\left(\mathrm{v}_{\mathrm{m}}\right)$. The quality of pattern separation was evaluated following the second presentation of the patterns (Fig. 5). By setting the matching threshold low $\left(\mathrm{v}_{\mathrm{m}}=0.7\right)$, no more learning could occur on the second trial; both patterns in a pair were found, according to the matching threshold, to be drawn to representations that were similar enough to the representations already stored on the first trial. Consequently, no significant difference was observed with respect to the results described above (ANOVA, $1 \mathrm{P}$ vs $2 \mathrm{P} ; \mathrm{F}_{19,180}=0.96, \mathrm{P}=0.51$ ). Using a higher threshold for matching $\left(\mathrm{U}_{\mathrm{m}}=0.95\right)$, only the pattern encoded by recruiting the inhibition circuit on the first trial showed an exact match on the second trial. Too often, at high input similarity, the other pattern in the pair could not satisfy the matching threshold, enabling learning to recur on the second trial. This is well illustrated in the results of Fig. 5C showing an increase in the number of HGC recruited after the second repetition of learning, suggesting that at high level of input similarity the representations of both patterns in a pair became mediated by the inhibition circuit. As a result, a significant improvement in pattern separation was observed after two learning trials (Fig. 5A). Specifically, there were main effects of both input similarity $\left(\mathrm{F}_{19,180}=59.85, \mathrm{P}<0.001\right)$ and number of trials $\left(\mathrm{F}_{1,180}=51.06, \mathrm{P}<0.001\right)$ with a significant interaction $\left(\mathrm{F}_{19,180}=6.13\right.$, $\mathrm{P}<0.001$ ). Simple effects analysis of this interaction showed that learning on the second trials had no effect on pattern separation for pairs with low similarities (all $\mathrm{P}>0.05$ for input similarity $<0.7$ ) while significantly improved pattern separation on pairs with higher similarities (all $\mathrm{P}<0.05$ ).

\section{Storage Capacity}

The observation that the S-I-O model can operate as a strong pattern separator, not only in the DG but also in CA3, suggests that this separation would have a significant impact on CA3 storage capacity as well. To substantiate this premise, the $\mathrm{S}$ and S-I-O models were trained to store a set of $\mathrm{N}$ input patterns, and then switched to recall mode in order to test their ability to retrieve the stored patterns (Fig. 6).

A significant difference was noted between the S and S-I-O models (Fig. 6A). Specifically, ANOVA revealed a significant main effect of model $\left(\mathrm{F}_{1,18182}=11863.67, \mathrm{P}<0.001\right)$, main effect of storage load $\left(\mathrm{F}_{8,18182}=827.68, \mathrm{P}<0.001\right)$ with a significant model $\times$ load interaction $\left(\mathrm{F}_{8,18182}=661.78, \mathrm{P}<0.001\right)$; post-hoc tests at each level of storage load $(\mathrm{N})$ revealed that both models were able to retrieve the stored patterns almost perfectly for $\mathrm{N} \leq 50$ (all $\mathrm{P}>0.05$ ); but as the number of stored patterns increased further, storage capacity significantly plummeted in the $\mathrm{S}$ model but not in the S-I-O model (all $\mathrm{P}<0.001$ for $\mathrm{N}>50$ ). Fig. $6 \mathrm{~B}$ shows how orthogonalization was gradually engaged, along with inhibition, to come up with successful retrieval of the stored patterns in the S-I-O model. Up to $\mathrm{N}=150$, the maximum numbers of HGC/HCA3 were kept as default. We then wanted to see how the model would perform if more cells were available. We found (Fig. 6A and B, gray shaded area) that the S-I-O model was able to maintain its performance as the number of stored patterns increased to 175 or to 200, however, orthogonalization was engaged in the encoding of more than $80 \%$ of the additional stored patterns (HCA3=40.9 at $\mathrm{N}=150$ vs. 82.2 at $\mathrm{N}=200$ ). This is because the PPCA3 network seems to operate near saturation as $\mathrm{N}$ increases beyond 150; therefore, a high level of potential interference can occur more frequently. Although orthogonalization could improve performance under this condition, it is important to bear in mind that the functional significance of the process of orthogonalization goes beyond mere boost in storage capacity at near saturation in the model. To further emphasize this point, we increased $\theta_{\mathrm{h}}$, with respect to its default value to favor inhibition over orthogonalization. This could increase storage capacity up to $\mathrm{N}=200$ by recruiting orthogonalization only in response to extremely high interference (HCA3=37.4 for $\mathrm{N}=200$ ); however, this came at the price of a significant decrease in the quality of retrieval (ANOVA, main effect of model, $\mathrm{F}_{1,18182}=1399.18, \mathrm{P}<0.001$; post-hoc tests, all $\mathrm{P}<0.01$ for $\mathrm{N}>50$ ). This suggests that the default threshold values drive the behavior of the model in a way that resonates better with the proposed role for the orthogonalization circuit in the model.

In order to further quantify the specific contributions of inhibition and orthogonalization circuits, we repeated the above simulations with the S-I and S-O models. By contrasting the results in Fig. 6A, C and E, it can be seen that the S-I-O model performed much better than either of the reduced models; at the default values of MC thresholds, for example, retrieval performance was significantly higher (ANOVA, S-I-O vs. S-O, main effect, $\mathrm{F}_{1,18182}=440.62$, P<0.001; S-I-O vs. S-O, main 
effect, $\mathrm{F}_{1,18182}=3665.96, \mathrm{P}<0.001$; post-hoc tests, S-I-O vs. S-O and S-I-O vs. S-I: all $\mathrm{P}<0.01$ for $\mathrm{N} \geq 100$ ) while no significant increase was observed in the number of HCA3 recruited to deal with interference (ANOVA, S-I-O vs. S-O: P=0.5). At high storage load, the number of HGC recruited by the S-I model decreased slightly (ANOVA with post-hoc tests, S-I-O vs S-I: $\mathrm{P}<0.001$ for $\mathrm{N}>100$ ). This reflects an increase in interference when orthogonalization is compromised. The difference between inhibition and orthogonalization was more apparent when either circuit was allowed to be recruited for any level of interference (orthogonalization with $\theta_{\mathrm{h}}=0$, Fig. 6C; inhibition with $\theta_{\mathrm{l}}=0$ and $\theta_{\mathrm{h}}=1$, Fig. $6 \mathrm{E}$ ). In this latter case, the S-O model provided optimal retrieval performance, whereas the S-I model could not retrieve all stored patterns correctly because inhibition reduced but did not completely suppress interference among the stored patterns. It is also worth noting from the results shown in Fig. 6C-F that varying the number of HGC/HCA3 up or down from default values does not systematically affect the performance of the model at fixed threshold values. That is to say, for the same number of stored patterns, increasing the number of HCA3 is not sufficient to increase recall accuracy unless a concomitant decrease in $\theta_{\mathrm{h}}$ is made to favor recruitment of orthogonalization at lower levels of interference (Fig. 6C and D).

\section{SIMULATION OF EMPIRICAL DATA}

\section{The Involvement of the DG in Encoding and Retrieval Processes}

On a theoretical basis, Rolls and Treves have argued that the mossy fiber input to CA3 may be necessary for memory encoding but not for retrieval; the latter may be initiated by the direct perforant path input to CA3 (Treves and Rolls, 1994; Rolls, 2013). However, empirical evidence appears to be mixed regarding the involvement of the DG during recall; some studies suggest that the DG is only needed for encoding new memories (Lassalle et al., 2000; Lee and Kesner, 2004), while others suggest a role in both encoding and retrieval (Gilbert et al., 2001; McHugh et al., 2007; Hunsaker et al., 2008; Goodrich-Hunsaker et al., 2008; Hunsaker et al., 2008; Daumas et al., 2009; Nakashiba et al., 2012). This discrepancy might potentially reflect differences in task conditions. Where the task is trial-unique, behavioral impairments are mostly observed under conditions of subtle discrimination (Gilbert et al., 2001) or high emotional salience (Daumas et al., 2009).

In (Lassalle et al., 2000), selective blockade of mossy fibers exhibited learning deficits on a spatial learning task in the Morris water maze, but had no effect on recall. In contrast, short-term memory retrieval deficits were produced by immediate post-training blockade of mossy fibers in contextual fear conditioning tasks (Daumas et al., 2004, 2009). These results could be reasonably taken to suggest that the hippocampus, with DG mossy fibers inactivated, might have failed in consolidating learning at CA3 synapses in contextual fear conditioning but not in spatial learning tasks. Based on our model, an alternative account would be that the DG-CA3 network invokes distinct circuits for memory encoding in these two specific aversive tasks. Beside the level of input similarity with previously stored memories, the level of emotional arousal induced by the experience may play a key part in determining which circuitry needs to be recruited during memory encoding of a specific experience. Specifically, we assume that sparsification can be associated with learning under lowarousal conditions, as seems to be the case in the spatial task since the water temperature was warm (Lassalle et al., 2000); since learning occurs at the EC-CA3 and CA3-CA3 synapses, the non-engagement of the DG following post-training inactivation of mossy fibers should not affect retrieval. On the other hand, an increased demand on pattern separation may be triggered under conditions of relatively high arousal, as is the case for fear conditioning, and therefore, learning might rely substantially more on the DG than on CA3. Consistent with this prediction is evidence demonstrating enhancing effects of arousal on pattern separation (Segal et al., 2012; Leal et al., 2014). Interestingly, this enhancement appears to be mediated by an increase in norepinephrine activity during encoding (Segal et al., 2012). This suggests that the inactivation of mossy fibers by diethyldithiocarbamate in the spatial learning task (Lassalle et al., 2000) might down-regulate the need for the DG to engage in pattern separation, while a compensatory increase of norepinephrine release may be induced by the aversive shock in fear conditioning tasks (Daumas et al., 2004), leading to normal or even increased load on pattern separation.

As another example, Gilbert et al. (2001) used a delayed-match-to-sample paradigm to test rats, with colchicine-induced lesions in their DG, for their one-trial memory of spatial locations. During the sample phase, rats were trained to displace an object, which covered 1 of 15 locations along a row of food wells, to receive a food reward and return to the start-box on a cheese-board maze. The rats were then required to choose between two objects identical to the sample phase object; one covered the same well as the sample phase object (correct choice), while the second object covered a different unbaited well along the row of food wells (incorrect choice). Five spatial separations, 15, 37.5, 60, 82.5, and $105 \mathrm{~cm}$, were randomly used to separate the correct object and the foil object during the choice phase. The results showed that control rats learn the task with $75 \%$ or better accuracy based on 80 trials, whereas DG-lesioned rats were significantly impaired at short and medium spatial separations $(15,37.5$, and $60 \mathrm{~cm})$ but their performance grew much better at large separations $(82.5$ and $105 \mathrm{~cm})$. 
Based on their results, Gilbert et al. (2001) argued that rats rely on distal environmental cues to differentiate between spatial locations, and that DG pattern separation computations should be critical when the two locations are close together because the overlap between distal cues would be high.

We replicated the spatial discrimination component of the task of Gilbert et al. (2001) in the model using a set of 25 pairs of input patterns, each pair having a similarity of $0.1,0.3,0.5,0.7$ or 0.9 . The number of pairs was equated across the five degrees of similarity. During encoding, all the pairs were presented randomly to the model (the paired patterns were presented sequentially) and the output patterns were recorded. Then the pairs were presented again to the model with the DG activated (control) or inactivated (lesion). The ability of the model to distinguish between the two patterns in each pair is assessed in terms of the similarity between the CA3 output responses (Fig. 7A). We also measured the retrieval performance in terms of the similarity between stored and recalled patterns (Fig. 7C). The results were grouped and averaged according to the similarity of the input patterns comprising the different pairs. Although this setup does not correspond exactly to the working memory component of the delayed-match-to-sample task, an argument could be made that the assessment of the discrimination ability can be linked to choice performance in the task; this is meant in the sense that a low similarity between the spatial representations of the target object and the foil can be presumed to reflect an ability to make a correct choice due to low probability of interference at the level of the place-reward associations that could be formed within or outside the hippocampus (Rolls, 2013; Kassab and Alexandre, 2015).

As can be shown in Fig. 7A, the model with the DG inactivated maintained its performance on pairs with low similarity but completely failed to separate input patterns with high similarity. A repeated-measures two-way ANOVA revealed a significant lesion $\times$ similarity interaction, $\mathrm{F}_{4,20}=101.40, \mathrm{P}<0.001$. Simple effects analysis of this interaction showed that the lesioned model performed as well as the control model on pairs with a similarity of $0.1(\mathrm{P}=0.7)$ or $0.3(\mathrm{P}=0.16)$ but performed significantly worse on pairs with higher similarities $(\mathrm{P}<0.01)$. This is because the inactivation of DG-mediated inhibition, which is used only occasionally to overcome a very low risk of interference on pairs with low similarity (Fig. 7B), does not prevent the retrieval of the stored patterns but may decline the quality of retrieval, an effect that begins to be clearer on pairs with medium similarity. On pairs with high similarity, orthogonalization is used more frequently (Fig. 5B). In this case, the inactivation of DG blocks access to the representations of the orthogonalized patterns; this is why the lesioned model retrieved the representation of the other pattern in the pair, instead (Fig. 7A and C).

\section{Morphing Environments}

In another approach, electrophysiological recordings have been used to track changes in hippocampal activity patterns during exposure to different environments that were gradually morphed between two familiar environments with different shapes, a square and a circle. The study by Wills et al. (2005) showed that, for small morphs, CA3 activity patterns are attracted to either of the preestablished representations of the square or the circle. In contrast, Leutgeb et al. (2007) simultaneously recorded DG and CA3 principal cells and reported a gradual transition in both DG and CA3 activity patterns along the morph sequence; however, DG was much more sensitive to small morphs than CA3.

To show how the model addresses these data, a sequence of seven binary patterns with $10 \%$ activity were constructed by linearly decreasing similarity through each pattern from the first to the last (Fig. 8). As in (Leutgeb et al., 2007), Patterns 1 and 7 were initially stored in the model. Then we tested three different scenarios with the morph patterns. In the first, learning was disabled in the model, and then, all the patterns were presented in sequence and the DG and CA3 outputs were recorded. As can be shown in Fig. 8, DG and CA3 responded very differently; DG exhibited a large decrease in the similarity between its responses to a stored pattern and a small morph (Patterns 1 to 2 or to 3), while, for small morphs, CA3 activity patterns were aligned with one of the stored patterns, making a rather sharp transition similar to the one found in Wills et al. (2005). We did not report the results obtained with the $S$ model because learning of the patterns 1 and 7 occurred through sparsification in the S-I-O model, and thus, no significant difference was found between the two models.

In the next two scenarios, we consider both models and learning was allowed to occur if the retrieved pattern in CA3 does not match any of the previously-stored patterns according to a low or a high threshold for matching, $\mathrm{v}_{\mathrm{m}}$.

With a low threshold, $\mathrm{v}_{\mathrm{m}}=0.7$, learning occurred most often for intermediate morphs, in both the $\mathrm{S}$ model and the S-I-O model (Fig. 9A and B, respectively). Learning new representations for intermediate morphs decreased their similarity to CA3 representations for the two end shapes. Furthermore, since sparsification/inhibition based mechanisms, which tended to dominate in the S-I-O model (Fig. 9C), do not guarantee an absolute separation between CA3 representations, small 
morphs had a stronger tendency to retrieve a mixture of stored representations in CA3. This caused gradual transition in the population activity of CA3, in a way that accords well with findings of Leutgeb et al. (2007). It is worth stressing, however, that the model would not produce such a transition if the EC input representations for intermediate shapes were biased towards that of either of the two end shapes, as it appears to be the case in (Wills et al., 2005).

With the matching threshold increased, $\mathrm{v}_{\mathrm{m}}=0.95$, learning could occur even for small morphs. This further linearized transitions in the S model due to increased interference from stored representations (Fig. 9A), while it made small morphs more likely to be detected in the S-I-O model (Fig. 9B). As a result, a significant drop in the similarity between CA3 representations was observed in the S-I-O model $\left(\mathrm{F}_{1,5}=21.27, \mathrm{P}<0.01\right)$, simply due to an increase in the recruitment of orthogonalization/inhibition based circuits during learning of small morphs (Fig. 9D). It is also possible to additionally reduce this similarity to almost zero with a higher value for $\mathrm{v}_{\mathrm{m}}$ and/or lower values for $\mathrm{MC}$ thresholds.

\section{DISCUSSION}

\section{The Conceptual Framework}

The present work offers a novel perspective on how the DG and CA3 are engaged during the encoding of episodic memories. Our account suggests that (1) distinct circuits in the DG-CA3 network provide potential substrates for distinct mechanisms of pattern separation with different degrees of robustness against interference; (2) The hilus acts as a site of convergence for various inter- and intra-hippocampal signals mediating the selection of the most appropriate circuitry for a given situation; (3) The controlling influence of these signals reflect interactions between bottom-up factors, such as pattern similarity, and top-down factors, such as motivation, which together determine the required level of pattern separation; (4) The selection of circuitry derives from a trade-off between the level of pattern separation required and network resources. This account is instantiated in a simplified, biologically constrained, model of the DG-CA3 network. The model identifies three potential circuits underlying three pattern separation mechanisms: sparsification, inhibition, and orthogonalization.

The primary circuit performs a sparse coding in which only a small proportion of the granule cells are active for any given episode. This sparse coding is governed by feedback inhibition without any learning-related changes to keep in with the rapid, single-trial acquisition of episodic memory. Despite claims that sparseness would be the most efficient way to encode episodic memories, our simulation results, as other relevant studies (Weisz and Argibay, 2009; Myers and Scharfman, 2011), clearly demonstrate that this is not always the case, at least at the computational level. When the single-trial criterion is met, sparseness does not allow for optimal pattern separation behavior in response to high overlap in EC inputs (cf. Fig. 3), and this directly impinges on the storage capacity of CA3 (cf. Fig. 6). The two other circuits are, therefore, introduced to support sparsification.

In both of these latter circuits, learning takes place in the granule cell-mossy cell-granule cell loop allowing a physically separate population of granule cells (HGC) to form a second-level representation of EC inputs. A high overlap in input could cause an overlap in the primary population (PPGC) that carries the original, sparse representation of EC inputs and, consequently, an overlap in PPCA3 as well. In such a case, however, HGC can keep this overlap from affecting CA3 storage and recall in one of two ways. In the inhibition circuit, HGC can block out interference from previously stored memories. This strategy protects the stored memory from proactive interference but is likely to increase retroactive interference as the same system (PPCA3) is used for storage and recall (cf. Fig. 4, 5 and 6E). Thus, inhibition could improve the quality of retrieved memories but would not guarantee successful retrieval of all the stored patterns, especially at high levels of interference. In cases where both types of interference should be eradicated, HGC can provide excitatory drive to a spatially separate population of CA3 pyramidal cells (HCA3), setting up a complete orthogonal representation for the memory to be stored more effectively in CA3. The simulation results show that pattern separation could be maximized (cf. Fig. 4) and optimal retrieval could be achieved relying solely on orthogonalization (cf. Fig. 6E).

It is important to underscore that orthogonalization is expected to contribute to pattern separation only if necessary because CA3 pyramidal cells are limited in number when compared with dentate granule cells. This expectation is consistent with existing empirical evidence showing that pattern separation is not always optimal (Yassa and Stark, 2011; Leutgeb et al., 2007; Deng et al., 2013). In the morphing experiments of Leutgeb et al. (2007), for example, a nearly linear decrease was observed in the correlation between CA3 population responses as morphing increased. While our model was able to produce similar results (cf. Fig. 9), it could also further significantly improve discrimination between small morphs, primarily by increasing the matching threshold to favor learning, and secondarily by decreasing $\mathrm{MC}$ thresholds to favor 
inhibition/orthogonalization over sparsification. We speculate that absolute discrimination between morphs was not empirically seen due to the lack of any emotional, motivational, or attentional modulation in the morphing environments. It would be reasonable to expect dramatically different results if the animal's attention was directed to specific morphs, e.g., via reward and fear-conditioning procedures. In line with this speculation, a recent electrophysiological study tested mice during exploratory food-motivated running in two highly similar boxes; however, unlike Leutgeb et al. (2007), two different kinds of food rewards were offered in the different boxes: cereal (low-value reward) and chocolate (high-value reward) (Senzai and Buzsáki, 2017). In CA3, most cells were found to be active in one or the other box, while a significant overlap was observed in the DG. These results can be explained in terms of a change in the animal's internal state which might be induced by the difference in reward values associated with the two boxes and assume that this change might modulate pattern separation in a way that greater attention is given to subtle differences in environmental states.

It was also evident from the results (cf. Fig. 3, 4 and 6) that a better performance-resource trade-off would be brought with both inhibition and orthogonalization circuits included in the model, rather than with either one alone. The choice among circuits is associated with the integration of intrinsic and extrinsic signals which come to keep the DG informed about the required level of pattern separation and the level of network resources. Such signals are likely to dynamically regulate the activity of mossy cells either directly or indirectly via neuromodulatory effects, as also suggested in previous studies (Scharfman, 2007; Myers and Scharfman, 2009; Scharfman, 2016), rendering them more or less likely to be recruited during learning and, thus, allowing a more or less elaborated encoding to occur in the DG-CA3 network.

\section{Potential Neural Substrates and Functional Significance}

Crucially the known details of the DG-CA3 circuitry provide compelling support to the conceptual framework we propose for the organization of DG/CA3 principal cells into functionally distinct circuits. Here we emphasize the heterogeneity of the DG/CA3 cells in terms of several important, albeit often neglected, anatomical and physiological properties and describe how they may be related to the proposed model.

Although mature granule cells show great similarity in their morphological and intrinsic electrophysiological properties, their activity is regulated by highly heterogeneous inhibitory circuits (Amaral et al., 2007; Freund and Buzsáki, 1996). Particularly relevant is the effect of perforant path stimulation on granule cells which has been shown to be non-uniform in that only a fraction of granule cells are responsive to stimulation, regardless of its intensity (Dieni et al., 2013; Yu et al., 2013); this effect was attributed to differential inhibition on granule cells because all the cells were able to respond when inhibition was blocked. Asymmetry has also been identified in the ratio of inhibitory neurons across the DG (e.g. there are twice as many basket cells in the suprapyramidal blade as in the infrapyramidal blade (Seress and Pokorny, 1981)), and related to differences in the recruitment of granule cells located in the two blades of the DG at different septotemporal levels (Scharfman et al., 2002; Alme et al., 2010; Yu et al., 2013). It is unclear, however, how various subtypes of GABAergic neurons contribute to the inherently limited responsiveness of granule cells.

Our model raises the possibility that the activity of granule cells might be differentially controlled by local interneurons, with axons impinging precisely on the distal two-thirds of granule cell dendrites (Freund and Buzsáki, 1996), thereby preventing dendritic integration of EC excitation in a subset of granule cells and favoring their activation by their inputs from mossy cells. The intrinsic integrative properties of granule cell dendrites may also account for different modes of input integration and further attenuation of distal inputs (Krueppel et al., 2011). Accordingly, granule cells may be more easily excited by afferent inputs from either the EC or MC, leading to two independent populations of granule cells, similar to those included in the model, PPGC and HGC. Perisomatic inhibition might provide a more specific mechanism to control competition among the first population (PPGC) in response to the EC drive, thereby ensuring sparse activity, whereas dendritic inhibition keeps the second population (HGC) silent except under certain circumstances where they can become active concomitantly with PPGC.

Based on the currently available data, we consider HIPP cells, especially a subset with dendrites in the molecular layer, as the most likely candidate for this inhibitory effect, as they are activated upon perforant path stimulation with a latency shorter than granule cells (Scharfman, 1991), and appear to be innervated by direct monosynaptic input from the EC (Deller et al., 1996). This implies that inhibition is likely to precede and potentially dominate EC monosynaptic excitation of granule cells. In an alternative, not necessarily mutually exclusive, perspective, inputs other than direct EC projections might be able to activate HIPP cells prior to encoding to ensure adequate temporal dynamics. These inputs could be from dentate glutamatergic and GABAergic neurons, direct cholingeric and GABAergic inputs from the septum, and from deep 
layer EC neurons which preferentially target GABAergic neurons in the inner molecular, the granule cell layer, and the hilus (Deller et al., 1996; Freund and Buzsáki, 1996; Amaral et al., 2007). Especially dentate glutamatergic neurons which show persistent activation, such as semilunar granule cells (Larimer and Strowbridge 2010) and mossy cells (Duffy et al., 2013), might provide direct ongoing basal depolarizing input to hilar GABAergic neurons. It is further notable that HIPP cells are not preferentially targeted by CA3 backprojections (Buckmaster et al., 1996; Wittner et al., 2006; Fig. 1), suggesting functional specialization of hilar interneurons.

The model is also compatible with the possibility that HGC, or at least a fraction thereof, are adult-born neurons. Even before they reach complete maturation, newborn neurons receive their first glutamatergic innervations from mossy cells (Chancey et al., 2014) and exhibit functional connectivity with CA3 after 2-3 weeks post-mitosis (Toni et al., 2008), and therefore, can be functionally integrated at early stages in both inhibition and orthogonalization circuitry. This view is also strongly consistent with substantial experimental evidence supporting a pivotal role for adult neurogenesis in pattern separation (Clelland et al., 2009; Nakashiba et al., 2012).

In CA3, the two spatially segregated populations of the model, PPCA3 and HCA3, have close correspondences with pyramidal cells in the distal and proximal portions of CA3, CA3a,b and CA3c, respectively (Ishizuka et al., 1990; Li et al., 1994; Scharfman, 2007; Witter, 2010). Cells in the distal portion have, like PPCA3, extensive recurrent collaterals that dominate locally within CA3, whereas proximal cells, like HGC, have relatively weak local connectivity and short apical dendrites that do not reach the stratum lacunosum-moleculare and, therefore, almost devoid of the direct monosynaptic input from the EC. The lack of direct EC innervation is compensated for by an increased density of mossy fiber input: In proximal CA3, mossy fibers are distributed deep to, within, and superficial to the pyramidal cell layer, making "double" terminal fields upon apical and basal dendrites of the pyramidal cells in what have been called the suprapyramidal and infrapyramidal mossy fiber bundles. The infrapyramidal bundle greatly decreases at distal CA3 where all mossy fibers tend to extend in the stratum lucidum parallel to the pyramidal cell layer. Also CA3c is the major source of CA3 collateralization in the hilus (Scharfman, 2007). Altogether these anatomical features indicate that only distal CA3 pyramidal cells, and by analogy PPCA3 in the present model, are compatible with an autoassociative memory function with a dual input for encoding and retrieval; the remaining cells at proximal parts, and by analogy HCA3 in the model, might subserve these two processes differently and largely under the control of mossy fiber afferents.

Given the distoproximal increasing gradients of CA3 backprojection (Li et al., 1994; Scharfman, 2007; Witter, 2010), the actions of CA3 pyramidal cells on hilar cells might not be directly exerted by distal cells (PPCA3) but by disynaptic activation via CA3c cells. It is also important to note that it is, thus far, unclear whether CA3 pyramidal cells may directly excite mossy cells (although indirect evidence exists, e.g. Scharfman,1994), suggesting that CA3 backprojection actions may be polysynaptically mediated, or at least facilitated, by direct interaction with the septum or other structures.

Also of related interest is the finding that granule cells roughly follow a topographic organization in their projections to CA3, with cells in the suprapyramidal blade preferentially projecting to distal CA3, and cells in the infrapyramidal blade preferentially projecting to proximal CA3 (Witter, 2010). Along the same lines, Galimberti et al. (2010) demonstrated that mossy fibers exhibit significant structural plasticity that proceeds according to similar topographic principles. Also the axons of newborn neurons preferentially (albeit not exclusively) contribute to the infrapyramidal mossy fiber bundle (Römer et al., 2011), whose size has been linked early on with better hippocampal-dependent learning capabilities (Crusio and Schwegler, 2005). It may be, therefore, useful to entertain the possibility that PPGC and HGC might not be uniformly dispersed along the DG blades. Specifically, PPGC might be more present in the suprapyramidal blade where GABAergic interneurons are more numerous and more densely innervated by mossy fibers (Seress and Pokorny, 1981; Ribak and Peterson, 1991), whereas the orthogonalization action on CA3 might mainly be exerted by granule cells in the infrapyramidal blade via their direct projections on the proximal basal dendrites of CA3c. It is also tempting to think of the two mossy fiber inputs in CA3c in terms of an economy drive to use CA3c pyramidal cells. When CA3c cells are driven by apical inputs alone, they may fire at low firing rates and can be considered as being part of PPCA3 during sparsification/inhibition. On the other hand, when CA3c cells are driven by additional inputs on their proximal basal dendrites, they may fire at high firing rates as being part of HCA3 during orthogonalization. Such firing rate encoding has been observed empirically (Leutgeb et al., 2007); however, our prediction is that the change in CA3 firing rates may be a result of using different pattern separation mechanisms, instead of being a pattern separation mechanism per se. Yet, it is important to stress that any conclusion in this respect should be treated cautiously due to differences among strains and species (Crusio and Schwegler, 2005). 


\section{Predictions and Experiments}

It is apparent from the preceding section that our model represents a radical departure from traditional single-population models, especially in that it can account for why retrieval deficits are observed under certain experimental circumstances but not others following post-training lesions of the DG (e.g., Gilbert et al. (2001); Daumas et al. (2009)). While our account holds the general understanding that the DG has the ability to amplify differences in input patterns through sparsification, it goes further by suggesting that the DG, under conditions of high load on pattern separation, learns to boost discrimination via additional mechanisms: inhibition or orthogonalization. Whenever either of these two mechanisms is involved in learning, DG lesion will lead to deficits in memory retrieval but for different reasons.

Because inhibition simply acts to prevent irrelevant pyramidal cells from responding to input from the direct perforant pathway, lesioning DG does not abolish retrieval per se but leads to a decline in the quality of retrieval due to an inability to trigger the inhibition-mediated discriminatory mechanism that has been set up in the DG. Alternatively, when orthogonalization is engaged during learning, the indirect perforant input via the DG (EC-DG-CA3) provides the only pathway to reach the orthogonalized memory representations in CA3, and therefore, a complete failure in retrieval will be produced by DG lesions.

Much evidence from previous studies can be taken as preliminary support of this prediction. For instance, a positive correlation was observed between memory precision and learning-induced increases in mossy fiber terminals onto CA3 interneurons (Ruediger et al., 2011; Restivo et al., 2015). It is plausible that adult-born granule cells heavily account for this correlation as they have significantly more contacts with CA3 interneurons than mature granule cells (Restivo et al., 2015) and silencing their outputs does affect pattern separation in CA3 (Clelland et al., 2009; Nakashiba et al., 2012). Whether these observations vary along the proximodistal axis has not been explored, however.

In keeping with the anatomical guidelines we have outlined above, our model would predict, more precisely, that: 1) memory precision depends on DG-mediated inhibition of distal CA3 where direct innervation by EC afferents is strong; 2) As we presume that mossy fiber transmission to CA3 pyramidal cells is inactivated during retrieval, this inhibition is more likely to be mediated by interneurons projecting to the distal apical dendrites of CA3 pyramidal cells, such as O-LM (Freund and Buzsáki, 1996); 3) the increased inhibition acts to reduce, not to cancel, excitation in distal CA3. On the other hand, when a complete loss of memory follows DG lesions, our model predicts a net shutdown of excitation in proximal CA3, but not necessarily in distal CA3; activity in distal CA3 could be observed especially if the orthogonalized memory overlaps with memories stored in distal CA3. It is worth noting that this latter prediction depends on two assumptions that warrant consideration. First, we presume within the current model that proximal CA3 has the ability to suppress activity in distal CA3 via inhibitory control. Still, we do not rule out a potential role for mossy fiber inputs in this inhibitory control on distal CA3; empirical work will be needed to disentangle these two possibilities. Second, our model does not include direct projections from PPCA3 to HCA3 (which may be, as evoked above, the first element of a disynaptic pathway from CA3 to the hilus, but also from CA3 to CA1), but it should be anticipated that, in vivo, distal CA3 could elicit excitation in proximal CA3, especially if the orthogonalized memory overlaps with memories stored in distal CA3. Testing the latter prediction experimentally may, therefore, require blockade of synaptic transmission at CA3 recurrent connections; one way to avoid this additional manipulation is to identify experimental scenarios in which pattern separation occurs via orthogonalization for other reasons than similarity.

The emotional and motivational effects on pattern separation and hippocampal neural representations have been demonstrated empirically (Kennedy and Shapiro, 2009; Nakashiba et al., 2012; Segal et al., 2012; Leal et al., 2014), though to a far lesser extent than pattern similarity. In this regard, it is intriguing that the hilus receives afferents from all neuromodulatory systems (Amaral et al., 2007), notably noradrenergic afferents from the locus coeruleus which are much denser than anywhere else in the hippocampus (Harley, 2007). These systems signal important environmental aspects such as novelty, uncertainty, salience and risks, and thus are in position to exert strong influence on memory, learning, and behavior. Our model predicts that these kinds of neuromodulatory signals occur either together or separately and act in concert with cortical inputs to keep the DG informed about the changing demands on pattern separation under changing circumstances. Specifically, we have demonstrated via the model that CA3 can detect memory interference arising from pattern similarity (cf. Fig. 9; Leutgeb et al., 2007) or spatial overlap in the external environment (cf. Fig. 7; Gilbert et al., 2001). With increasing levels of similarity, CA3 feedback excitation of mossy cells increases and leads to increased discriminability from other memories. At the same time, we have demonstrated by varying MC thresholds (cf. Fig. 3 and 6) that other extrahippocampal signals that converge to the hilus can modulate the activity of hilar mossy cells, either directly 
or indirectly through their effects on hilar interneurons. Consistent with this prediction is evidence for selective norepinephrine modulation of mossy cells and different types of hilar interneurons (Bijak and Misgeld, 1995) and for norepinephrine-mediated enhancing effects on pattern separation (Segal et al., 2012; Leal et al., 2014).

A somewhat similar prediction was made by previous studies (Myers and Scharfman, 2009, 2011; Jinde et al., 2012) but the engagement of hilar cells was suggested to modulate sparsity of activity in the DG through their drive onto DG granule cells. Our account differs both computationally and functionally, at least, in three fundamental aspects. First, we assume that a major function of mossy cells is to integrate signals they receive and to accordingly shuttle the control between distinct pathways that underlie the DG's engagement in pattern separation. This assumption, however, is not mutually exclusive with previous suggestions which are relevant to the sparsification mechanism included in the present model. Second, we assume that hilar interneurons, especially HIPP cells, may prevent a subpopulation of granule cells from responding to EC afferents through their inhibitory action on distal dendrites of granule cells. The diversity of afferent inputs to HIPP cells, however, suggest that a more complex circuitry may contribute to this inhibitory function to ensure adequate network dynamics. It is also worth noting that the loss of this dendritic inhibition in the proposed model can entail a strong increase of excitability with the majority of HGC being activated, and thus can account for granule cell hyperexcitability that follows hilar cell loss, including mossy cells and inhibitory interneurons (Jinde et al., 2012), in a way quite different from the currently available hypotheses (Scharfman, 2016). Third, we assume heterogeneity in mossy cell responsiveness to CA3 backprojection, possibly due to differential CA3-mediated inhibition on these cells. Precisely how mossy cells act in response to CA3 input remains to be determined empirically.

Another point to consider is related to the interpretation of experimental observations. Previous experiments involving electrophysiological recordings have suggested that overlapping populations of granule cells participate in multiple representations of individual memories (Leutgeb et al., 2007; Alme et al., 2010), while other studies, using immediate-early gene activation, have shown that distinct granule cell populations encode similar but non-identical memories with relatively limited overlap (Chawla et al., 2005; Deng et al., 2013). This discrepancy is part of what makes the DG's function in pattern separation very puzzling. It is fair, however, to mention that appealing explanations have been proposed but, rather, in terms of a single functional population, or functionally independent populations, of granule cells. Alternatively, based on our model, these findings can be revisited in terms of multiple functional populations of granule cells that can be simultaneously brought into activity under certain conditions. Specifically, we speculate that high overlap can be observed in the DG in two different situations with potentially high level of interference due to similarity. In situations with high attentional or motivational loads, as discussed earlier in reward or fear-conditioning experiments, input similarity can be detected in CA3 and lead to the recruitment of HGC which becomes simultaneously active with PPGC; overlap in PPGC, which is expected to occur due to input similarity, could be associated with the overlap that was empirically observed in the DG. In this case, however, no, or at least much less, overlap should be expected, after learning, in CA3. In other situations, as discussed in the morphing experiments, encoding may never occur or occur through sparsification alone; in this latter case, increased overlap should be expected in the DG but also, to a similar or even greater extent, in CA3.

Crucially, based on the analysis above, inference can be made about which circuit of the model is activated by specifying the regional variations in overlap in response to different experiences; sparsification can be inferred if overlap is low or high in both DG and CA3, while other circuits are more likely if overlap is higher in DG than it is in CA3. Especially in this latter case, granule cells should not be lumped together; instead, potential subpopulations have to be individually targeted depending on whether they show unique or overlapping responses. Our account suggests that granule cells with experiencespecific responses, even if significantly less numerous, would be the key players in the successful separation in CA3. Of course, this possibility remains speculative but, hopefully, points to new directions to design more detailed experiments characterizing functional differences in potential subpopulations of granule cells.

\section{Conclusion}

The present work enriches current perspectives on how pattern separation might function throughout the DG-CA3 network. The hypothesis that distinct circuits coexist and contribute differentially to this process makes sense of various experimental observations that have, thus far, defied explanations at both computational and biological levels. The existing evidence, while compatible with the hypothesis, is still preliminary and much work remains to be done to validate the present model and identify actual circuits and mechanisms. The anatomical details and the various experimentally testable predictions we have presented in this paper would, hopefully, help move forward in this direction. 
It should be noted that our model is simplified, for the purpose of clarity, in a way meant to only capture fundamental elements of our hypothesis as a whole. It is the focus of our ongoing work to improve both the biological and functional significances of the model, especially by considering the dynamics of newborn neurons in the DG and by integrating additional relevant extra- and intrahippocampal structures to explore in more detail their modulatory effects on pattern separation. Also, of potentially great interest for future investigations is to check whether the coexistence of distinct DGCA3 circuits mediating pattern separation emerges from evolutionary or developmental expansions (Treves et al., 2008).

\section{Compliance with Ethical Standards}

Conflict of Interest The authors declare that they have no conflict of interest.

Ethical approval This article does not contain any studies with human participants or animals performed by any of the authors.

\section{REFERENCES}

Acsády, L., Kamondi, A., S k, A., Freund, T., and Buzsáki, G. (1998), GABAergic cells are the major postsynaptic targets of mossy fibers in the rat hippocampus, J Neurosci., 18, 9, 3386-3403

Aimone, J. B., Wiles, J., and Gage, F. H. (2009), Computational influence of adult neurogenesis on memory encoding, Neuron, 61, 2, 187-202

Alme, C., Buzzetti, R., Marrone, D., Leutgeb, J., Chawla, M., Schaner, M., et al. (2010), Hippocampal granule cells opt for early retirement, Hippocampus, 20, 10, 1109-23

Aloisi, A., Casamenti, F., Scali, C., and Carli, G. P. G. G. (1997), Effects of novelty, pain and stress on hippocampal extracellular acetylcholine levels in male rats, Brain Res., 748, 1-2, 219-26

Amaral, D. G., Scharfman, H. E., and Lavenex, P. (2007), The dentate gyrus: fundamental neuroanatomical organization (dentate gyrus for dummies), Prog Brain Res., 163, 3-22

Amari, S. (1989), Characteristics of sparsely encoded associative memory, Neural Networks, 2, 6, 451-457

Andersen, P., Bliss, T. V. P., and Skrede, K. K. (1971), Lamellar organization of hippocampal excitatory pathways, Exp. Brain Res., 13, 2, 222-238

Bakker, A., Kirwan, C. B., Miller, M., and Stark, C. E. (2008), Pattern separation in the human hippocampal CA3 and dentate gyrus, Science, 319, 5870, 1640-2

Berron, D., Schütze, H., Cardenas-Blanco, A. M. A., Kuijf, H. J., Kumaran, D., and Düzel, E. (2016), Strong evidence for pattern separation in human dentate gyrus., J Neurosci., 36, 29, 7569-79

Bijak, M., and Misgeld, U. (1995), Adrenergic modulation of hilar neuron activity and granule cell inhibition in the guineapig hippocampal slice, Neuroscience, 67, 3, 541-50

Bliss, T. V. and Lomo, T. (1973), Long-lasting potentiation of synaptic transmission in the dentate area of the anaesthetized rabbit following stimulation of the perforant path, J Physiol, 232, 2, 331-356

Buckmaster, P. S. and Schwartzkroin, P. A. (1994), Hippocampal mossy cell function: a speculative view, Hippocampus, 4, 4, 393-402

Buckmaster, P. S., Wenzel, H. J., Kunkel, D. D., and Schwartzkroin, P. A. (1996), Axon arbors and synaptic connections of hippocampal mossy cells in the rat in vivo, J Comp Neurol., 366, 2, 271-92

Chancey, H. J., Poulsen, D. J., Wadiche, J. I., and Overstreet-Wadiche, L. (2014), Hilar mossy cells provide the first glutamatergic synapses to adult-born dentate granule cells, Journal of Neuroscience, 34, 6, 2349-2354

Chawla, M. K., Guzowski, J. F., Ramirez-Amaya, V., Lipa, P., Hoffman, K. L., Marriott, L. K., et al. (2005), Sparse, environmentally selective expression of Arc RNA in the upper blade of the rodent fascia dentata by brief spatial experience, Hippocampus, 15, 5, 579-586

Clelland, C., Choi, M., Romberg, C., Clemenson, G. J., Fragniere, A., Tyers, P., et al. (2009), A functional role for adult hippocampal neurogenesis in spatial pattern separation, Science, 325, 5937, 210-3

Crusio, W. E. and Schwegler, H. (2005), Learning spatial orientation tasks in the radial-maze and structural variation in the hippocampus in inbred mice, Behav Brain Funct., 1, 3 
Daumas, S., Ceccom, J., Halley, H., Francés, B., and Lassalle, J.-M. (2009), Activation of metabotropic glutamate receptor type 2/3 supports the involvement of the hippocampal mossy fiber pathway on contextual fear memory consolidation, Learn Mem., 16, 8, 504-7

Daumas, S., Halley, H., and Lassalle, J.-M. (2004), Disruption of hippocampal CA3 network: effects on episodic-like memory processing in c57bl/6j mice, European Journal of Neuroscience, 20, 2, 597-600

de Almeida, L., Idiart, M., and Lisman, J. E. (2007), Memory retrieval time and memory capacity of the CA3 network: role of gamma frequency oscillations, Learn Mem, 14, 11, 795-806

Deller, T., Martinez, A., Nitsch, R., and Frotscher, M. (1996), A novel entorhinal projection to the rat dentate gyrus: direct innervation of proximal dendrites and cell bodies of granule cells and GABAergic neurons, J. Neurosci., 16, 10, 3322--33

Deng, W., Mayford, M., and Gage, F. H. (2013), Selection of distinct populations of dentate granule cells in response to inputs as a mechanism for pattern separation in mice, eLife, 2, e00312

Dieni, C., Nietz, A. K., Panichi, R., Wadiche, J., and Overstreet-Wadiche, L. (2013), Distinct determinants of sparse activation during granule cell maturation, J Neurosci., 33, 49, 19131-42

Duffy, A. M., Schaner, M. J., and J. Chin J, a. H. E. S. (2013), Expression of c-fos in hilar mossy cells of the dentate gyrus in vivo, Hippocampus, 23, 8, 649-55

Eichenbaum, H. (2004), Hippocampus: cognitive processes and neural representations that underlie declarative memory, Neuron, 44, 1, 109-20

Freund, T. F. and Buzsáki, G. (1996), Interneurons of the hippocampus, Hippocampus, 6, 4, 347-470

Galimberti, I., Bednarek, E., Donato, F., and Caroni, P. (2010), Epha4 signaling in juveniles establishes topographic specificity of structural plasticity in the hippocampus, Neuron, 65, 5, 627-42

Ge, S., Yang, C. H., Hsu, K. S., Ming, G. L., and Song, H. (2007), A critical period for enhanced synaptic plasticity in newly generated neurons of the adult brain, Neuron, 54, 4, 559-66

Gilbert, P. E., Kesner, R. P., and Lee, I. (2001), Dissociating hippocampal subregions: double dissociation between dentate gyrus and ca1, Hippocampus, 11, 6, 626-36

Gluck, M. A., Meeter, M., and Myers, C. E. (2003), Computational models of the hippocampal region: linking incremental learning and episodic memory, 7, 6, 269-276

Goodrich-Hunsaker, N. J., Hunsaker, M. R., and Kesner, R. P. (2008), The interactions and dissociations of the dorsal hippocampus subregions: how the dentate gyrus, CA3, and CA1 process spatial information, Behav Neurosci., 122, 1, 16-26 Harley, C. W. (2007), Norepinephrine and the dentate gyrus, Prog Brain Res., 163, 299-318

Hasselmo, M. E., Bodelón, C., and Wyble, B. P. (2002), A proposed function for hippocampal theta rhythm: Separate phases of encoding and retrieval enhance reversal of prior learning, Neural Comput., 14, 4, 793-817

Hasselmo, M. E., Wyble, B. P., and Wallenstein, G. V. (1996), Encoding and retrieval of episodic memories: Role of cholinergic and GABAergic modulation in the hippocampus, Hippocampus, 6, 6, 693-708

Hetherington, P. A., Austin, K. B., and Shapiro, M. L. (1994), Ipsilateral associational pathway in the dentate gyrus: an excitatory feedback system that supports N-methyl-D-aspartate-dependent long-term potentiation, Hippocampus, 4, 4, 42238

Hunsaker, M. R., Rosenberg, J. S., and Kesner, R. P. (2008), The role of the dentate gyrus, CA3a,b, and CA3c for detecting spatial and environmental novelty, Hippocampus, 18, 10, 1064-73

Ishizuka, N., Weber, J., and Amaral, D. G. (1990), Organization of intrahippocampal projections originating from ca3 pyramidal cells in the rat, J Comp Neurol., 295, 4, 580-623

Jackson, M. B., Scharfman, H. E. (1996), Positive feedback from hilar mossy cells to granule cells in the dentate gyrus revealed by voltage-sensitive dye and microelectrode recording, J Neurophysiol., 76, 1, 601-16

Jinde, S., Zsiros, V., Jiang, Z., Nakao, K., Pickel, J., Kohno, K., et al. (2012), Hilar mossy cell degeneration causes transient dentate granule cell hyperexcitability and impaired pattern separation, Neuron, 76, 6, 1189-200

Kassab R., and Alexandre F. (2015), Integration of exteroceptive and interoceptive information within the hippocampus: a computational study. Front Syst Neurosci., 5, 9, 87

Kee, N., Teixeira, C., Wang, A., and Frankland, P. (2007), Preferential incorporation of adult-generated granule cells into spatial memory networks in the dentate gyrus, Nat Neurosci., 10, 3, 355-62

Kennedy, P. J., and Shapiro, M. L. (2009), Motivational states activate distinct hippocampal representations to guide goaldirected behaviors. Proc. Natl. Acad. Sci. U.S.A., 106, 10805-10810 
Kleschevnikov, A. M., and Routtenberg, A. (2003), Long-term potentiation recruits a trisynaptic excitatory associative network within the mouse dentate gyrus, Eur J Neurosci., 17, 12, 2690-702

Knoblauch, A., Palm, G., and Sommer, F. T. (2010), Memory capacities for synaptic and structural plasticity, Neural Computation, 22, 2, 289-341

Krueppel, R., Remy, S., and Beck, H. (2011), Dendritic integration in hippocampal dentate granule cells, Neuron, 71, 3, 512-28

Larimer, P. and Strowbridge, B. W. (2008), Nonrandom local circuits in the dentate gyrus, J Neurosci., 28, 47, 12212-23

Larimer, P. and Strowbridge, B.W. (2010), Representing information in cell assemblies: persistent activity mediated by semilunar granule cells, Nat Neurosci., 13, 2, 213-22

Lassalle, J. M., Bataille, T., and Halley, H. (2000), Reversible inactivation of the hippocampal mossy fiber synapses in mice impairs spatial learning, but neither consolidation nor memory retrieval, in the Morris navigation task, Neurobiol Learn Mem., 73, 3, 243-57

Leal, S.L., Tighe, S.K., Jones, C.K., and Yassa, M.A. (2014), Pattern separation of emotional information in hippocampal dentate and CA3, Hippocampus, 24, 9, 1146-55

Lee, I., Hunsaker, M., and Kesner, R. (2005), The role of hippocampal subregions in detecting spatial novelty, Behav Neurosci., 119, 1, 145-53

Lee, I. and Kesner, R. P. (2004), Encoding versus retrieval of spatial memory: double dissociation between the dentate gyrus and the perforant path inputs into CA3 in the dorsal hippocampus, Hippocampus, 14, 1, 66-76

Leutgeb, J. K., Leutgeb, S., Moser, M.-B., and Moser, E. I. (2007), Pattern separation in the dentate gyrus and ca3 of the hippocampus, Science, 315, 5814, 961-966

Li, X., Somogyi, P., Ylinen, A., and Buzsáki, G. (1994), The hippocampal ca3 network: an in vivo intracellular labeling study, J Comp Neurol., 339, 2, 181-208

Lisman, J. E. (1999), Relating hippocampal circuitry to function: Recall of memory sequences by reciprocal dentateCA3 interactions, Neuron, 22, 2, 233-42

Lopez-Rojas, J. and Kreutz, M. R. (2016), Mature granule cells of the dentate gyrus - passive bystanders or principal performers in hippocampal function, Neuroscience \& Biobehavioral Reviews, 64, 167-174

Lysetskiy, M., Földy, C., and Soltesz, I. (2005), Long- and short-term plasticity at mossy fiber synapses on mossy cells in the rat dentate gyrus, Hippocampus, 15, 6, 691-6

Marr, D. (1969), A theory of cerebellar cortex, J Physiol, 202, 2, 437-470

Marr, D. (1971), Simple memory: a theory for archicortex, Philosophical Transactions of the Royal Society of London. Series B, Biological Sciences, 262, 841, 23-81

McBain, C. J. (2008), Differential mechanisms of transmission and plasticity at mossy fiber synapses, Prog Brain Res., 169, 225-240

McHugh, T., Jones, M., Quinn, J., Balthasar, N., Coppari, R., Elmquist, J., et al. (2007), Dentate gyrus nmda receptors mediate rapid pattern separation in the hippocampal network, Science, 317, 94-99

McNaughton, B. L. and Nadel, L. (1990), Hebb-Marr networks and the neurobiological representation of action in space. In Gluck, M. A. and Rumelhart, D. E. (eds), Neuroscience and Connectionist Theory (Hillsdale, NJ: L. Erlbaum), pp 1-64

McNaughton, B.L., Barnes, C.A., Mizomori, S.Y., Green, E.J., and Sharp, P.E. (1991). The contribution of granule cells to spatial representation in hippocampal circuits: A puzzle. In: Morrell F. (ed), Kindling and Synaptic Plasticity: The Legacy of Graham Goddard. Boston:Springer-Verlag. pp 110-123

Morris, R. G. M. (2001), Episodic-like memory in animals: psychological criteria, neural mechanisms and the value of episodic-like tasks to investigate animal models of neurodegenerative disease, Philos Trans R Soc Lond B Biol Sci., 356, 1413, 1453-1465

Moser, E. I. And Moser, E. I. (2003), One-shot memory in hippocampal CA3 networks, Neuron, 38(2):147-8

Myers, C. E. and Scharfman, H. E. (2009), A role for hilar cells in pattern separation in the dentate gyrus: A computational approach, Hippocampus, 19, 4, 321-337

Myers, C. E. and Scharfman, H. E. (2011), Pattern separation in the dentate gyrus: A role for the CA3 backprojection, Hippocampus, 21, 11, 1190-215

Nakashiba, T., Cushman, J. D., Pelkey, K. A., Renaudineau, S., Buhl, D. L., McHugh, T. J., et al. (2012), Young dentate granule cells mediate pattern separation, whereas old granule cells facilitate pattern completion, Cell, 149, 1, 188-201 
O’Reilly, R. C. and McClelland, J. L. (1994), Hippocampal conjunctive encoding, storage, and recall: Avoiding a trade-off, Hippocampus, 4, 6, 661-82

O’Reilly, R. C. and Rudy, J. W. (2001), Conjunctive representations in learning and memory: Principles of cortical and hippocampal function, Psychological Review, 108, 2, 311-345

Restivo, L., Niibori, Y., Mercaldo, V., Josselyn, S. A., and Frankland, P. W. (2015), Development of adult-generated cell connectivity with excitatory and inhibitory cell populations in the hippocampus, Journal of Neuroscience, 35, 29, 10600-12

Ribak, C. E. and Peterson, G. M. (1991), Intragranular mossy fibers in rats and gerbils form synapses with the somata and proximal dendrites of basket cells in the dentate gyrus, Hippocampus, 1, 4, 355-64

Rolls, E. (2013), The mechanisms for pattern completion and pattern separation in the hippocampus, Frontiers in Systems Neuroscience, 7, 74

Rolls, E. T. and Treves, A. (1998), Neural networks and brain function. Oxford University Press, Oxford, New York

Römer, B., Krebs, J., Overall, R. W., Fabel, K., Babu, H., Overstreet-Wadiche, L., et al. (2011), Adult hippocampal neurogenesis and plasticity in the infrapyramidal bundle of the mossy fiber projection: I. co-regulation by activity, Front Neurosci., 5, 107

Ruediger, S., Vittori, C., Bednarek, E., Genoud, C., Strata, P., Sacchetti, B., et al. (2011), Learning-related feedforward inhibitory connectivity growth required for memory precision, Nature, 473, 7348, 514-8

Scharfman, H. E. (1991), Dentate hilar cells with dendrites in the molecular layer have lower thresholds for synaptic activation by perforant path than granule cells, J. Neurosci., 11, 6, 1660-73

Scharfman, H. E. (1994), Evidence from simultaneous intracellular recordings in rat hippocampal slices that area CA3 pyramidal cells innervate dentate hilar mossy cells, J. Neurophysiol., 72, 2167-80

Scharfman, H. E., (1995), Electrophysiological evidence that dentate hilar mossy cells are excitatory and innervate both granule cells and interneurons, J Neurophysiol, 74, 1, 179-94

Scharfman, H. E. (2007), The CA3 “backprojection” to the dentate gyrus, Prog Brain Res., 163, 627-37

Scharfman, H. E. (2016), The enigmatic mossy cell of the dentate gyrus, Nat Rev Neurosci., 17, 9, 562-575

Scharfman, H., Sollas, A., Smith, K., Jackson, M., and Goodman, J. (2002), Structural and functional asymmetry in the normal and epileptic rat dentate gyrus, J Comp Neurol., 454, 4, 424-39

Scoville, W. B. and Milner, B. (1957), Loss of recent memory after bilateral hippocampal lesions, Journal of Neurology, Neurosurgery and Psychiatry, 20, 1, 11-21

Segal, S.K., Stark, S.M., Kattan, D., Stark, C.E., and Yassa, M.A. (2012), Norepinephrine-mediated emotional arousal facilitates subsequent pattern separation, Neurobiol Learn Mem., 97, 4, 465-9

Senzai, Y. and Buzsáki, G. (2017), Physiological properties and behavioral correlates of hippocampal granule cells and mossy cells, Neuron, 93, 3, 691-704

Seress, L. and Pokorny, J. (1981), Structure of the granular layer of the rat dentate gyrus. a light microscopic and golgi study, J Anat, 133, Pt 2, 181-95

Toni, N., Laplagne, D., Zhao, C., Lombardi, G., Ribak, C., Gage, F., et al. (2008), Neurons born in the adult dentate gyrus form functional synapses with target cells, Nat Neurosci., 11, 8, 901-7

Treves, A. and Rolls, E. T. (1994), Computational analysis of the role of the hippocampus in memory, Hippocampus, 4, 3, 374-391

Treves, A., Tashiro, A., Witter, M., and Moser, E. (2008), What is the mammalian dentate gyrus good for?, Neuroscience, $154,4,1155-72$

Tulving, E. (1972), Episodic and semantic memory. In Tulving E. and Donaldson W. (eds) Organization of Memory. New York: Academic Press, pp 382-402

Vago, D. and Kesner, R. (2008), Disruption of the direct perforant path input to the ca1 subregion of the dorsal hippocampus interferes with spatial working memory and novelty detection, Behav Brain Res., 189, 2, 273-83

Weisz, V. I. and Argibay, P. F. (2009), A putative role for neurogenesis in neurocomputational terms: Inferences from a hippocampal model, Cognition, 112, 2, 229-240

West, M., Slomianka, L., and Gundersen, H. (1991), Unbiased stereological estimation of the total number of neurons in the subdivisions of the rat hippocampus using the optical fractionator, Anat Rec., 231, 4, 482-97 
Wills, T., Lever, C., Cacucci, F., Burgess, N., and O’Keefe, J. (2005), Attractor dynamics in the hippocampal representation of the local environment, Science, 308, 5723, 873-6

Willshaw, D. J., Buneman, O. P., and Longuet-Higgins, H. C. (1969), Non-holographic associative memory, Nature, 222, 5197, 960-962

Wiskott, L., Rasch, M., and Kempermann, G. (2006), A functional hypothesis for adult hippocampal neurogenesis: Avoidance of catastrophic interference in the dentate gyrus, Hippocampus, 16, 3, 329-43

Witter, M. P. (2010), Connectivity of the hippocampus. In Cutsuridis, V., Graham, B. P., Cobb, S., and Vida, I. (eds) Hippocampal Microcircuits: A Computational Modelers Resource Book. New York, NY: Springer, pp 5-26

Wittner, L., Henze, D.A., Záborszky, L., and Buzsáki, G. (2006), Hippocampal CA3 pyramidal cells selectively innervate aspiny interneurons., Eur J Neurosci., 24, 5, 1286-98

Yassa, M. A. and Stark, C. E. L. (2011), Pattern separation in the hippocampus, Trends Neurosci., 34, 10, 515-25

Yu, E., Dengler, C., Frausto, S., Putt, M., Yue, C., Takano, H., et al. (2013), Protracted postnatal development of sparse, specific dentate granule cell activation in the mouse hippocampus, J Neurosci., 33, 7, 2947-60

\section{APPENDIX - IMPLEMENTATIONAL DETAILS}

Our model includes networks for the DG and CA3 regions of the hippocampus (Fig. 2). The cell numbers are scaled down to approximately 1/1000 the size of the rat hippocampus (West et al., 1991; Witter, 2010): 1000 PPGC, 120 HGC and 120 MC in the DG; 300 PPCA3 and 50 HCA3 in CA3; and external inputs are provided by 200 neurons in the EC. The simulated neurons are simple points with continuous firing rates. The postsynaptic activity is computed as a function of the neuron's membrane potential which evolves according to the sum of all excitatory and inhibitory synaptic inputs the neuron receives. The computations proceed in discrete time steps during which the activity of a homogeneous population of neurons is updated synchronously.

\section{The DG Network}

All inputs from the EC cells to a PPGC $\mathrm{i}_{\mathrm{i}}$ are summed up to a membrane potential, $\mathrm{V}_{\mathrm{i}}$ :

$$
V_{i}(t)=\sum_{j} W_{i j} E C_{j}(t)-I_{P P G C}
$$

where $\mathrm{EC}_{\mathrm{j}}$ is the activity of the jth $\mathrm{EC}$ cell; $\mathrm{W}_{\mathrm{ij}}$ refers to the synaptic weights between EC and PPGC; $\mathrm{I}_{\mathrm{PPGC}}$ is the amount of tonic inhibition in the DG which has been held constant at 0.75 . At the beginning of each simulation run, $\mathrm{W}_{\mathrm{ij}}$ are initialized as random values drawn from a normal distribution with a mean of 1 and a standard deviation of 0.05 and are further normalized onto each PPGC.

Within each cluster, PPGC compete among themselves and only those receiving maximal excitation are driven to fire. The firing rate is given by:

$$
\operatorname{PPGC}_{i}(t)=\left\{\begin{array}{c}
\tanh \left(\sigma V_{i}(t)\right), \text { if } V_{i}(t)=\underset{k \in C}{\operatorname{argmax}}\left(V_{k}(t)\right) \\
0, \text { otherwise }
\end{array}\right.
$$

with $\sigma$ is a constant defining the slope of the hyperbolic function and set to 0.1 for all PPGC, $k$ is the index that labels PPGC belonging to the same cluster $\mathrm{C}$ as PPGC $\mathrm{C}_{\mathrm{i}}$.

In the hilus, the membrane potential of a $\mathrm{MC}_{\mathrm{i}}$ depends upon its two main inputs. The influence of PPGC input is computed in a way similar to that described for associative memories with binary neurons (Willshaw et al., 1969; Knoblauch et al., 2010):

$$
V_{i}^{(P P G C)}(t)=\left\{\begin{array}{c}
V_{i}=\sum_{j} W_{i j} P P G C_{j}(t), \text { if } V_{i} \geq \sum_{j} P P G C_{j}(t) \\
0, \text { otherwise }
\end{array}\right.
$$

The weights of synapses that connect PPGC and MC are initially set to zero, and updated during learning according to the “clipped” Hebbian learning (Willshaw et al., 1969; Knoblauch et al., 2010), which means that $\mathrm{W}_{\mathrm{ij}}$ is changed from 0 to 1 
when both presynaptic and postsynaptic cells are simultaneously active $\left(\mathrm{MC}_{\mathrm{i}}>0\right.$ and $\left.\mathrm{PPGC}_{\mathrm{j}}>0\right)$ while further co-activations do not induce further changes.

The influence of CA3 backprojection on MC membrane potential is approximated by the summed activity of the whole population of PPCA3:

$$
V_{i}^{(P P C A 3)}(t)=G\left(\tanh \left(\sigma \sum_{j} W_{i j} \operatorname{PPCA3}_{j}(t)\right)\right)
$$

Here we assume a fully connected projection from PPCA3 to MC with all synaptic weights, $\mathrm{W}_{\mathrm{ij}}$, are set to 1 ; $\sigma$ is set to 0.1 ; and G(.) is a dual-thresholding function which implicitly defines the influence of PPCA3 on MC. Specifically, G(V $\left.V_{i}\right)$ causes one of the high-threshold mossy cells, $\mathrm{MC}_{\mathrm{h}}$, to be active if $\mathrm{V}_{\mathrm{i}}>\theta_{\mathrm{h}}$; or activates one of the low-threshold mossy cell, $\mathrm{MC}_{\mathrm{l}}$, if $\theta_{1}<V_{i} \leq \theta_{h}$; otherwise, CA3 backprojection has no influence on MC. This function also assigns 1/0 binary states to free vs. already recruited MC to ensure that a MC would be recruited only if it is not part of other previously-established memory traces. The default values for MC thresholds, $\theta_{1}$ and $\theta_{\mathrm{h}}$, are set to 0.1 and 0.5 , respectively.

The firing rate of a $\mathrm{MC}_{\mathrm{i}}$ is then given by:

$$
M C_{i}(t)=\tanh \left(\sigma\left(V_{i}^{(P P G C)}(t)+V_{i}^{(P P C A 3)}(t)\right)\right)
$$

with $\sigma$ is set to 10. The synaptic weights between MC and HGC are set to 1 and HGC are assumed to show equal responses as their presynaptic partners $\left(\mathrm{HGC}_{\mathrm{i}}(\mathrm{t})=\mathrm{MC}_{\mathrm{i}}(\mathrm{t})\right)$.

\section{The CA3 Network}

The activity of PPCA3 cells is driven by multiple excitatory and inhibitory inputs. During learning, PPGC mossy fiber inputs function as detonators for their PPCA3 targets provided that the latter are not under active inhibition from HGC/HCA3. The firing rate of a PPCA3 is given by:

$$
\operatorname{PPCA}_{i}(t)=\left\{\begin{array}{c}
\tanh \left(\sum_{j} W_{i j} P P G C_{j}(t)\right), \text { if } I_{i}=0 \\
0, \wedge \text { otherwise }
\end{array}\right.
$$

With

$$
I_{i}=\sum_{j} Q_{i j} H G C_{j}(t)+\sum_{j} Z_{i j} H C A 3_{j}(t)
$$

Here, $\mathrm{W}_{\mathrm{ij}}$ refers to the synaptic weights of mossy fibers which are all equal to $1 ; \mathrm{I}_{\mathrm{i}}$ refers to the summed inhibitory action that HGC and HCA3 might trigger onto PPCA3; all the weights of inhibitory synapses between HGC and PPCA3, $\mathrm{Q}_{\mathrm{ij}}$, are initiated at 1 , while the weights of inhibitory synapses between HCA3 and PPCA3, $\mathrm{Z}_{\mathrm{ij}}$, are all initiated and maintained at 1 . Learning occurs in CA3 by modifying the synaptic weights of EC-PPCA3 and PPCA3-PPCA3 connections according to clipped Hebbian learning and by setting the synaptic weights of the inhibitory connections between co-active HGC and PPCA3 cells to zero.

During recall, PPGC inputs are disabled. PPCA3 receive excitatory drives from the perforant path and recurrent projections, but may also undergo strong inhibition from local interneurons; HCA3 are only responsive to drive from HGC:

$$
\operatorname{PPCA}_{i}(t)=\left\{\begin{array}{c}
\tanh \left(\sigma\left(\sum_{j} W_{i j}^{P P} E C_{j}(t)+\sum_{k} W_{i k}^{R C} \operatorname{PPCA}_{k}(t-1)-I_{P P C A 3}(t)\right)\right), \text { if } I_{i}=0 \\
0, \wedge \text { otherwise }
\end{array}\right.
$$

with

$$
I_{P P C A 3}=\mu\left(\sum_{j} E C_{j}(t)+\sum_{k} P P C A 3_{k}(t-1)\right)
$$


and

$$
H C A 3_{i}(t)=H G C_{i}(t)
$$

Again, $\mathrm{I}_{\mathrm{i}}$ refers to the amount of inhibition arising from HGC/HCA3 on PPCA3 $3_{\mathrm{i}} ; \quad W_{i j}^{P P}$ and $W_{i k}^{R C}$ are respectively the synaptic weights of EC-PPCA3 and PPCA3-PPCA3 connections; $\mathrm{I}_{\mathrm{PPCA}}$ is the amount of inhibition that depends on incoming activity from the EC and CA3 itself; $\mu$ is a constant which has been set to 0.2. The activity of PPCA3 is computed repeatedly until the network settles into a steady state or until a maximum of 10 iterations is reached.

\section{Figures}

Fig. 1 A: Schematic transverse section through the rodent hippocampus. Three of the major hippocampal subregions are shown: the dentate gyrus (DG), CA3 with its CA3a,b and CA3c subdivisions, and CA1. Cortical inputs reach the hippocampus via the perforant path projections from the entorhinal cortex (EC). The perforant path axons of EC cells terminate on distal dendrites of dentate granule cells (GC), forming the first stage in the trisynaptic hippocampal loop. In the second stage, mossy fiber axons of GC makes giant boutons that innervate thorny excrescences of CA3 pyramidal cells and significantly more filopodial contacts and small en passant boutons that innervate CA3 interneurons (Not shown in the figure; Acsàdy et al., 1998). Schaffer collateral axons of CA3 pyramidal cells then project to distal dendrites of CA1 pyramidal cells, which project back to the EC (both directly and indirectly via the subiculum), closing the loop. In addition to innervating the DG, perforant path axons form monosynaptic excitatory contacts with CA3 and CA1 cells. There are also multiple recurrent and feedback excitatory pathways. Most prominent are strong recurrent collaterals within CA3, especially in CA3a,b. Some CA3 pyramidal cells also tend to project back to the DG, especially those located in ventral CA3c (Ishizuka et al., 1990; Li et al., 1994), where they form synaptic contacts with non-principal dentate cells, including hilar glutamatergic mossy cells (MC) and GABAergic interneurons (Scharfman, 2007). Besides, hilar cells receive highly convergent inputs from GC and form multiple excitatory and inhibitory feedback loops through the axons they project back to GC and local interneurons (Buckmaster et al., 1996; Larimer and Strowbridge, 2008). B: Local circuitry of the DG. The granule cells (GC) have their somata in the granule cell layer (GCL), their dendrites arborize through the inner, middle and outer thirds of the molecular layer (IML, MML and OML), and their axons widely ramify within the hilus. The perforant path axons of EC cells form synapses with GC dentrites in the MML and OML, while MC axons typically arborize ipsilateral and contralateral to their cell body and terminate on GC dendrites in the IML (Scharfman, 2016). MC receive direct input from GC mossy fibers, and from CA3 pyramidal neurons that extent their axon collaterals back into the hilus (Ishizuka et al., 1990; Scharfman, 1994). The interneurons in the DG are diverse: IN represents perisomatic-targeting interneurons, such as basket cells and chandelier cells, with dendrites spanning all the three layers and axons primarily located in the GCL; MOPP (molecular layer perforant path-associated cell) are GABAergic cells with both dendritic and axonal trees being largely limited to MML and OML; HIPP (hilar perforant path-associated cell) are hilar cells with their dendrites being limited to the hilus and their axons ascending to MML and OML; HIN represents other GABAergic hilar interneurons (Freund and Buzsáki, 1996). MC selectively innervate hilar interneurons that also preferentially target MC (Larimer and Strowbridge, 2008). It is likely that the main targets of MC are aspiny interneurons which are also preferentially innervated by CA3 recurrent collaterals (Buckmaster et al., 1996; Wittner et al., 2006).

Fig. 2 Schematic of the proposed model of the DG-CA3 network. The model defines three distinct, yet intertwined, circuits to mediate different pattern separation mechanisms with increasing degrees of robustness against interference. 1) The sparsification circuit is similar to standard models of the DG-CA3 network. The EC input diverges onto a large subpopulation of granule cells, PPGC, which further increase the sparseness of DG representations through their competitive interactions mediated by local somatic or axo-axonic feedback GABAergic interneurons. The activity of PPGC reaches a large subpopulation of CA3 pyramidal cells, PPCA3, via mossy fibers, and forces new representations on these CA3 cells. 2) The inhibition circuit involves additional recurrent excitatory feedback from PPGC, through a subset of mossy cells $\left(\mathrm{MC}_{1}\right)$, to a smaller subpopulation of granule cells, HGC, that mediate learning-dependent inhibition on PPCA3. 3) The orthogonalization circuit extends the sparsification circuitry by recurrent excitatory feedback from PPGC, through a subset of mossy cells $\left(\mathrm{MC}_{\mathrm{h}}\right)$, to a smaller subpopulation of granule cells, HGC, that drive strong excitation on a small subpopulation of CA3 pyramidal cells, HCA3, which, in turn, drive strong inhibition on PPCA3. The circuit selection is associated with the integration of intrinsic and extrinsic signals which converge to the hilus, directly or indirectly from PPCA3, from the septum, and other neuromodulatory systems; as a result, either $\mathrm{MC}_{1}$ or $\mathrm{MC}_{\mathrm{h}}$ or none of them, can be activated during learning depending the required level of pattern separation. The architecture of the model in its most basic form is drawn in black (the sparsification circuit). How this basic architecture is extended to include the inhibition and 
orthogonalization circuits is indicated, respectively, by green- and blue-colored connections. Stable, non-modifiable synaptic connections in the model are shown as solid lines, while plastic synaptic connections are shown as solid lines interrupted by two slanted bars. Excitatory projections are indicated by arrows and inhibitory projections by lines terminating in a vertical bar.

Fig. 3 A: Pattern separation in the basic model (S) and the proposed model (S-I-O) in the DG and CA3 networks. The diagonal line depicts equal similarities at the input and at the output; pattern separation occurs if similarity between the output patterns falls below the line and the greater the distance below the line, the greater the effect of pattern separation. B: The number of HGC recruited by the inhibition circuit (I) and the number of HGC/HCA3 recruited by the orthogonalization circuit (O) in the S-I-O model. C and D: The differential contributions of inhibition and orthogonalization circuits using the S-I and S-O models under the default setting of MC thresholds $\left(\theta_{1}=0.1\right.$ and $\left.\theta_{\mathrm{h}}=0.5\right)$. The results of the $S$ model from $A$ are replotted here for ease of comparison. E: The number of HGC recruited by the inhibition circuit. F: The number of HGC/HCA3 recruited by the orthogonalization circuit.

Fig. 4 A: The individual contributions of the inhibition (I) and orthogonalization (O) circuits using the S-I and S-O models. In order to enable the activation of these circuits following any potential risk of interference, MC thresholds were set to $\theta_{1}=0$ and $\theta_{\mathrm{h}}=1$ in the S-I model, while they were set to $\theta_{\mathrm{l}}=\theta_{\mathrm{h}}=0$ in the S-O model. B: The number of HGC recruited by the inhibition circuit. C: The number of HGC/HCA3 recruited by the orthogonalization circuit.

Fig. 5 A: Pattern separation in the S-I model after one presentation (1P) of the paired patterns for learning purpose (results replotted from Fig. 4A), and after two presentations (2P) of the patterns, using a matching threshold $\mathrm{v}_{\mathrm{m}}$ of 0.7 and 0.95 . B: The total number of HGC recruited by the inhibition circuit during one (1P) and two (2P) learning trials using $\mathrm{u}_{\mathrm{m}}=0.7$. C: The total number of HGC recruited by the inhibition circuit during one (1P) and two (2P) learning trials using $\mathrm{u}_{\mathrm{m}}=0.95$.

Fig. 6 Effect of the number of stored patterns on retrieval performance measured as the average similarity between the stored and retrieved patterns. The gray-filled area shows model results obtained with the number of HGC and/or HCA3 exceeding the values specified as default in the architecture of the present model (HGC=120 and HCA3=50). A: Performance of the S model vs. the S-I-O model with MC thresholds set to default $\left(\theta_{1}=.1\right.$ and $\left.\theta_{\mathrm{h}}=.5\right)$ and increased values $\left(\theta_{\mathrm{l}}=.3\right.$ and $\left.\theta_{\mathrm{h}}=.9\right)$. B: The number of HGC recruited by the inhibition circuit (I) and the number of HGC/HCA3 recruited by the orthogonalization circuit (O). C: Comparison of performance of the S-O model for different values of $\theta_{\mathrm{h}}$. D: The number of HGC/HCA3 recruited by the orthogonalization circuit in the S-O model. E: Comparison of performance of the S-I model for different values of $\theta_{1}$ and $\theta_{\mathrm{h}}$. F: The number of HGC recruited by the inhibitory circuit in the S-I model. The results of the $\mathrm{S}$ model taken from $\mathrm{A}$ are replotted in $\mathrm{C}$ and $\mathrm{E}$ for ease of comparison.

Fig. 7 Model results from the simulation of the spatial pattern separation task of Gilbert et al. (2001). A: The average similarity between CA3 output responses to different pairs of input patterns. The model with the DG activated (control) showed markedly reduced similarity in CA3 compared with similarity that existed in the input patterns. With the DG inactivated (lesion), CA3 responses were highly correlated for pairs that were similar or highly similar at the input. B: The S-I-O model tended to rely on inhibition for pairs with low input similarities but more on orthogonalization for pairs with higher similarities at the input. C: Retrieval performance in terms of the similarity between stored and recalled patterns. Without the DG, the capacity of the model to retrieve stored patterns got progressively worse as the input similarity became higher.

Fig. 8 The results obtained by the S-I-O model in the first scenario used in the simulations of the morphing experiment of Leutgeb et al. (2007). Similarity of DG/CA3 population responses to morphing when learning was disabled on morphed patterns (Patterns 2 to 6). DG exhibited clear separation for small morphs while CA3 responses were clearly attracted to either of the two previously stored representations of Pattern 1 or 7 . Pattern 4 caused retrieval of a mixture of the two previously stored representations in CA3.

Fig. 9 Model results obtained in two different novelty-based learning scenarios used in the simulations of the morphing experiment of Leutgeb et al. (2007). A: In the S model, morphing induced a gradual decrease in the similarity of CA3 population responses with $\mathrm{u}_{\mathrm{m}}=0.7$, but CA3 pattern separation decreased by increasing $\mathrm{u}_{\mathrm{m}}$ to 0.95 . B: In the S-I-O model, morphing also induced a gradual decrease in the similarity of CA3 population responses with $\mathrm{v}_{\mathrm{m}}=0.7$, but CA3 pattern separation increased by increasing $\mathrm{v}_{\mathrm{m}}$ to 0.95 . C: With a low threshold, $\mathrm{v}_{\mathrm{m}}=0.7$, sparsification/inhibition based mechanisms were mostly used to learn a new CA3 representation for Pattern 4 . D: With a high threshold, $\mathrm{v}_{\mathrm{m}}=0.95$, learning occurred more frequently and orthogonalization/inhibition based mechanisms tended to dominate learning of small morphs. 
Fig. 1

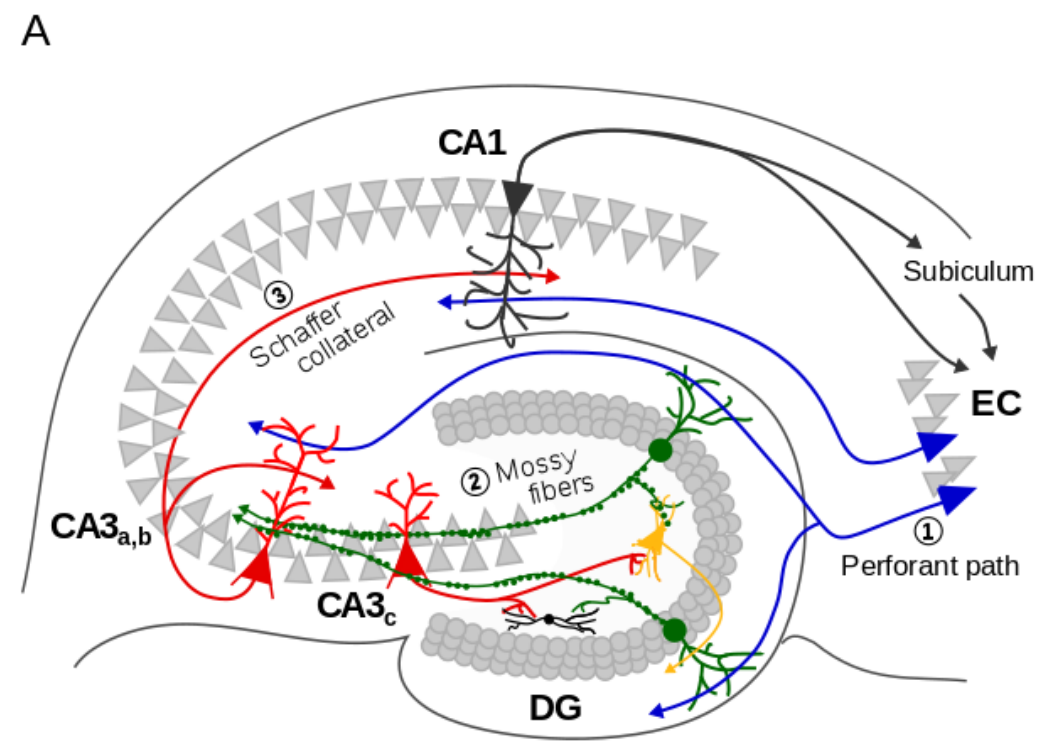

B

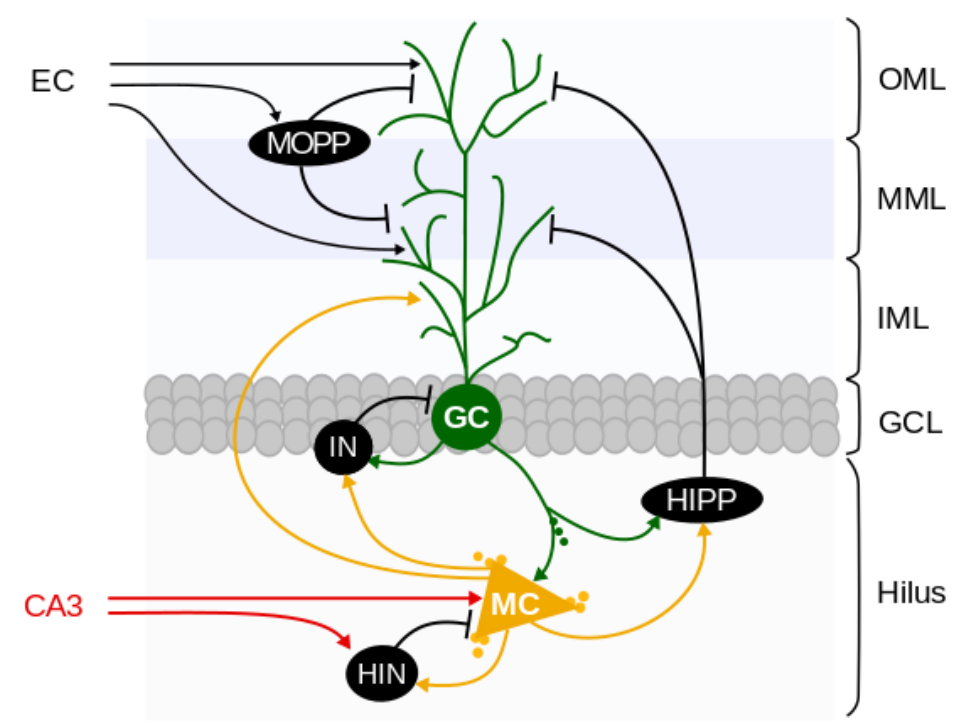


Fig. 2

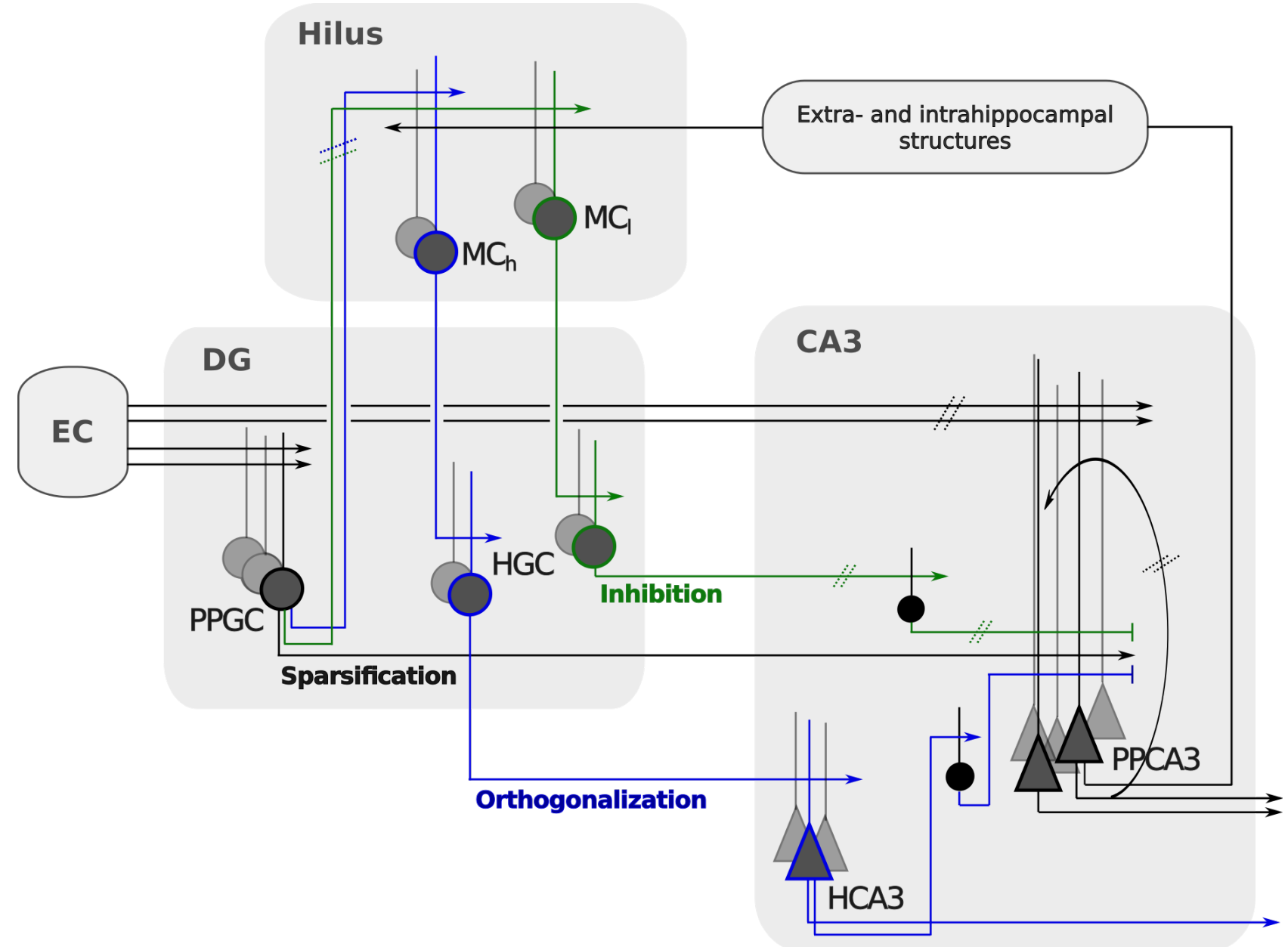


Fig 3
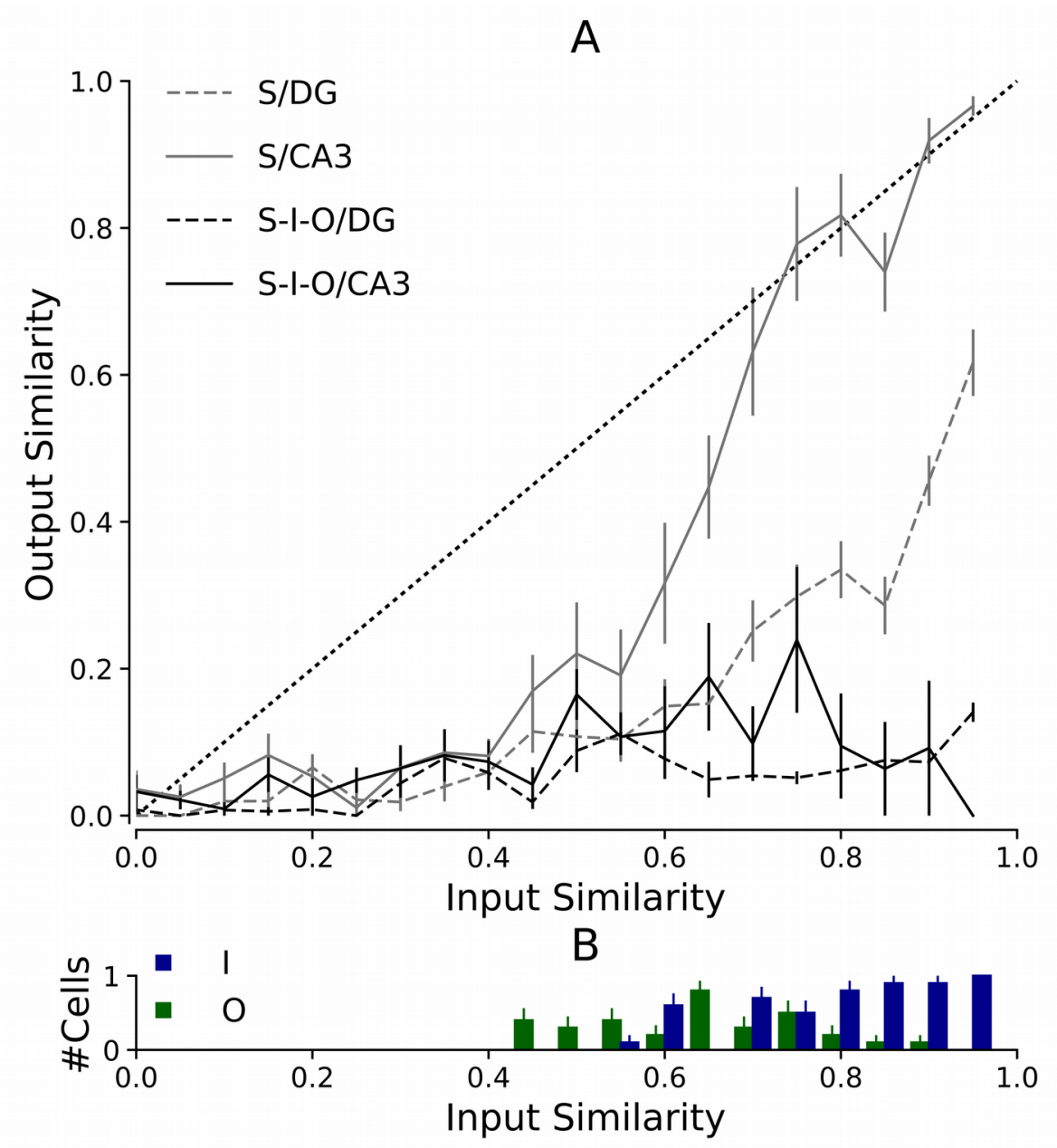

C
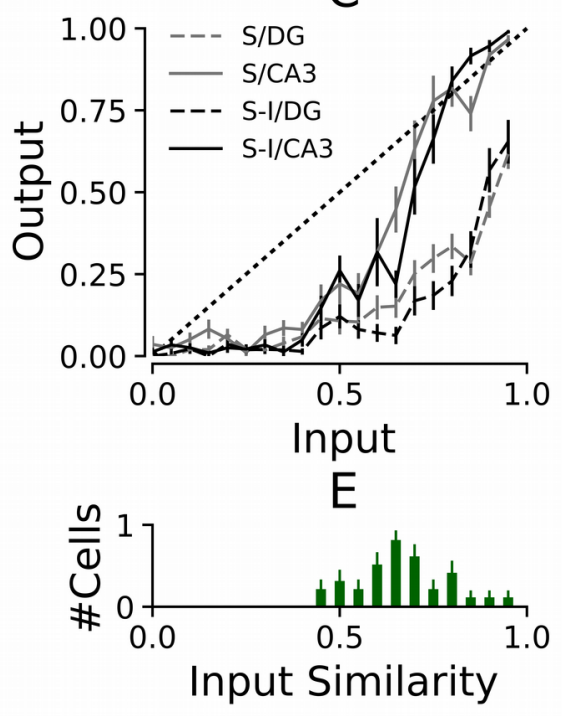

D
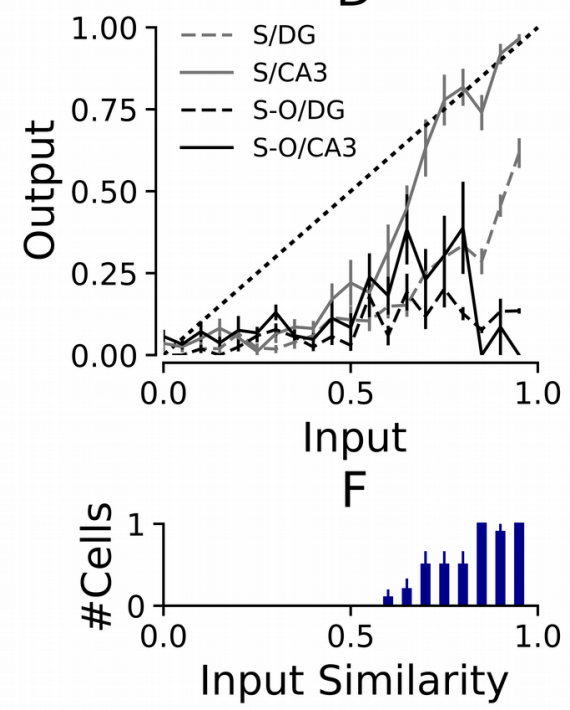
Fig 4
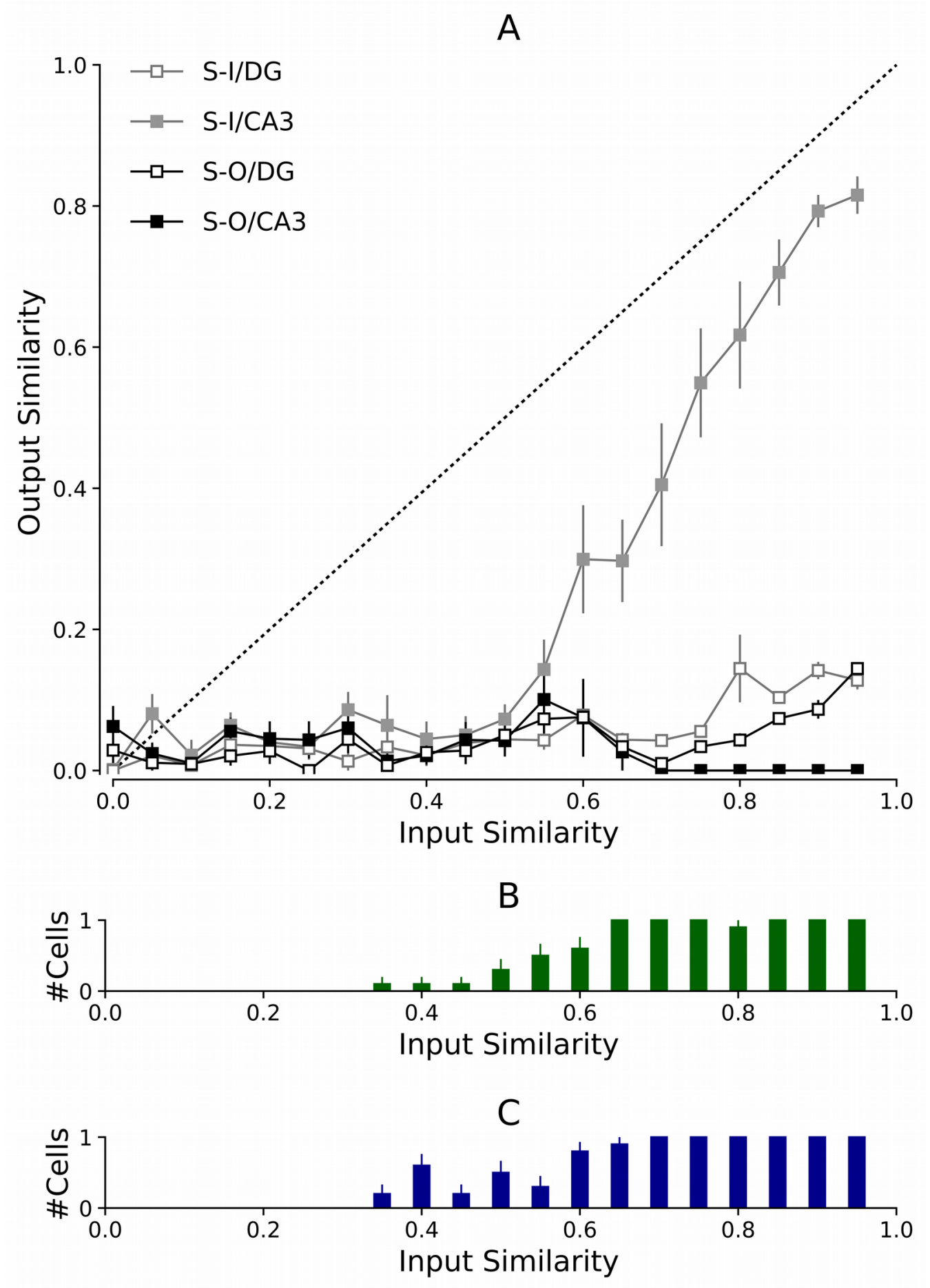
Fig 5
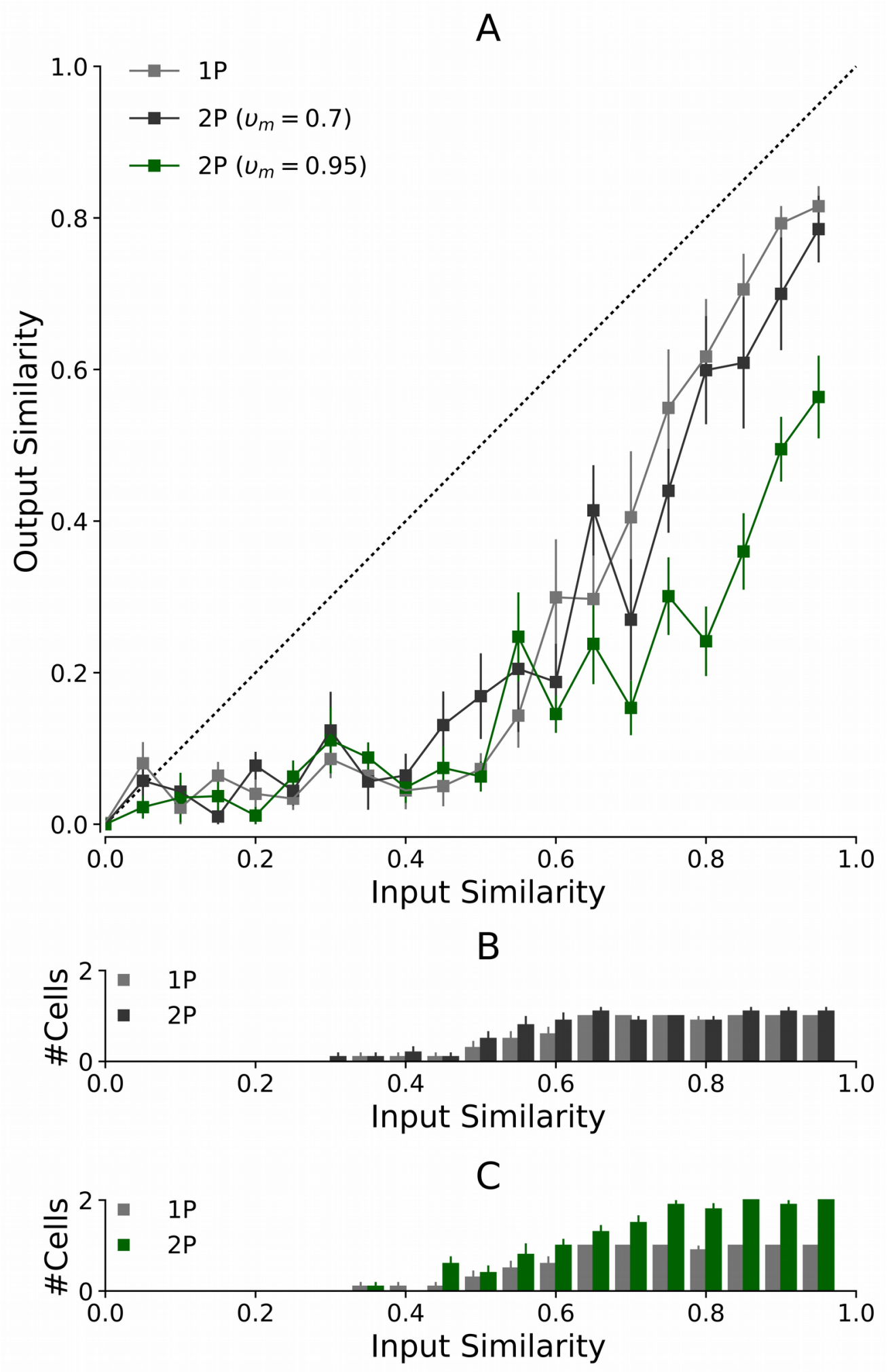
Fig 6
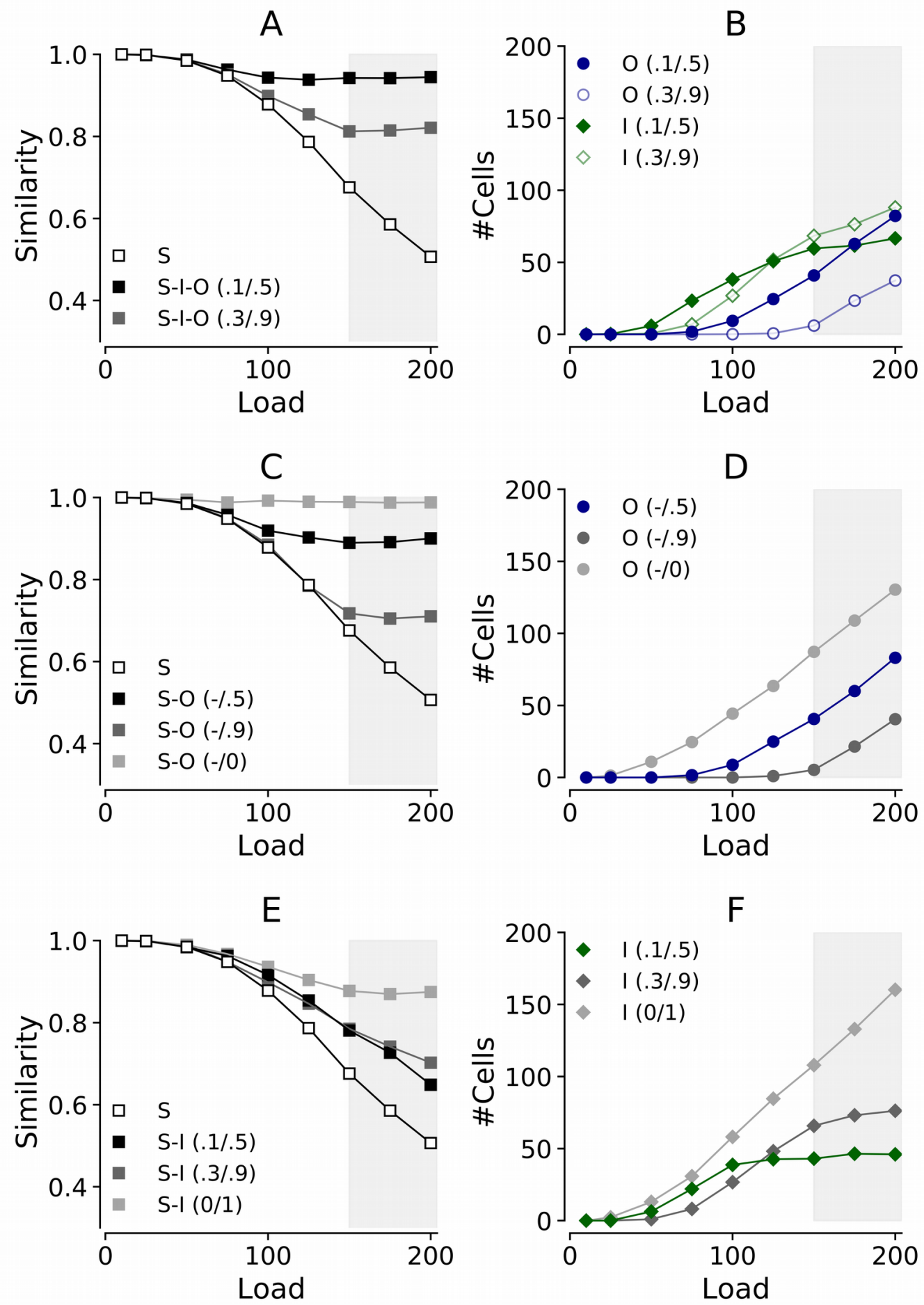
Fig 7

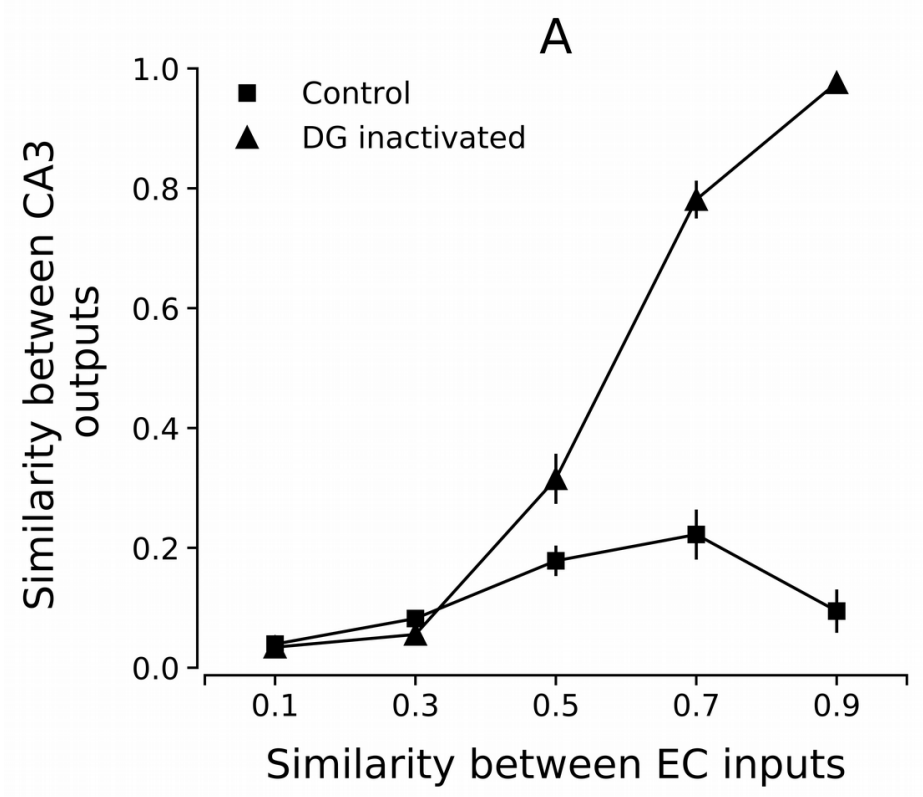

B

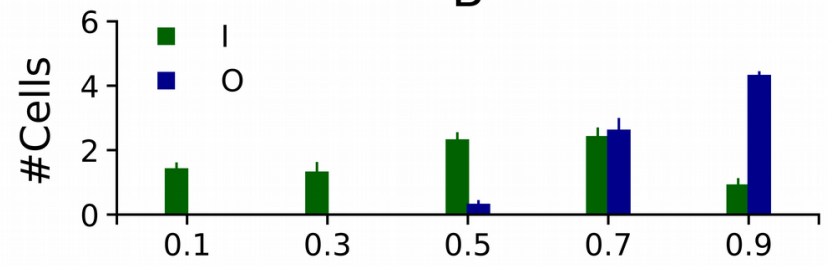

Similarity between EC inputs

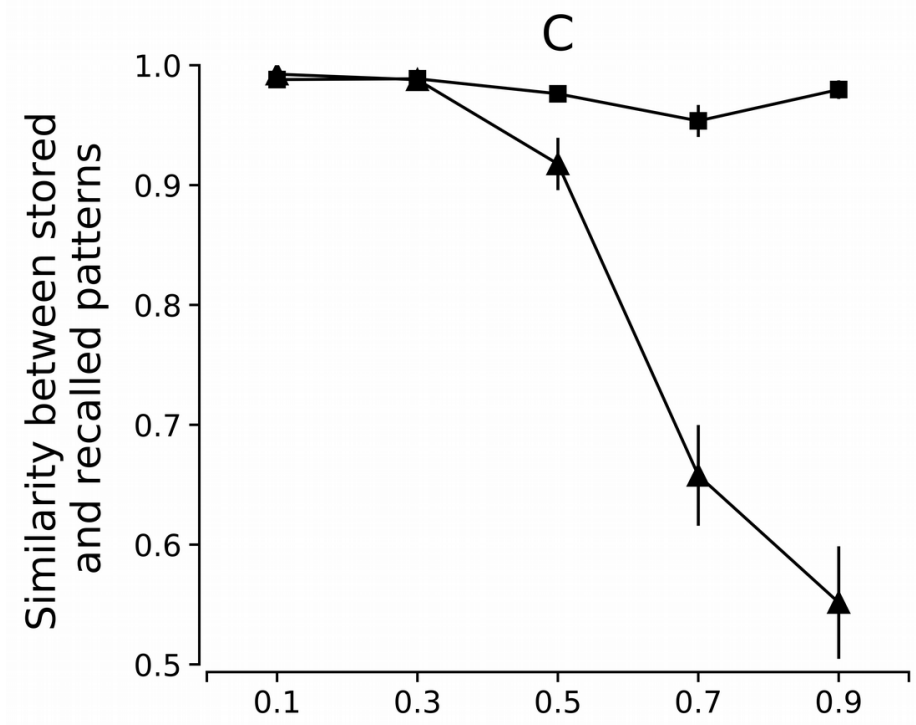

Similarity between EC inputs 
Fig. 8

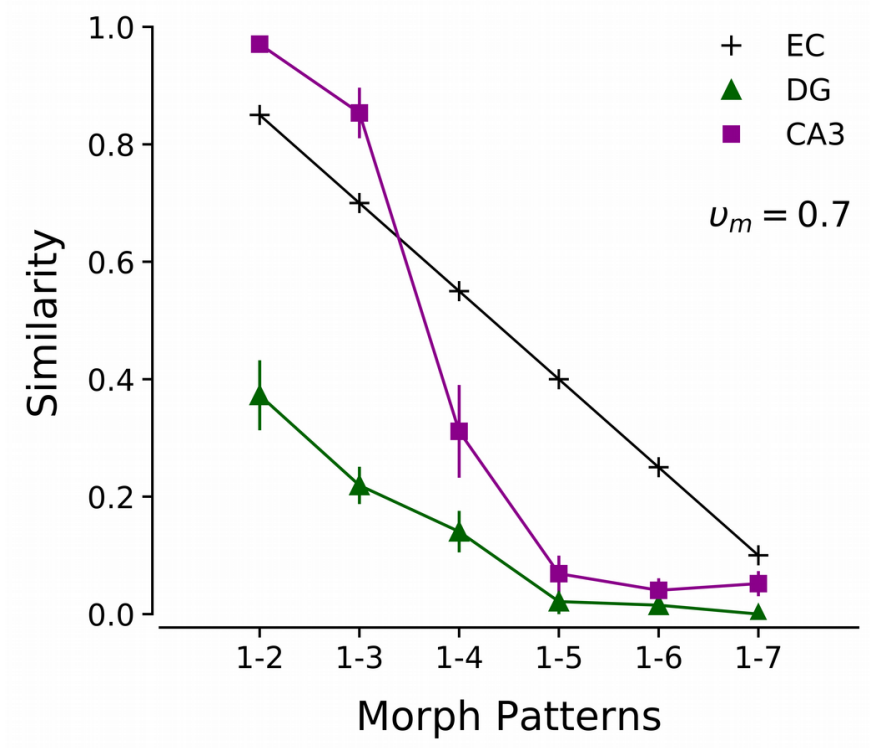


Fig. 9

A

The $\mathrm{S}$ model

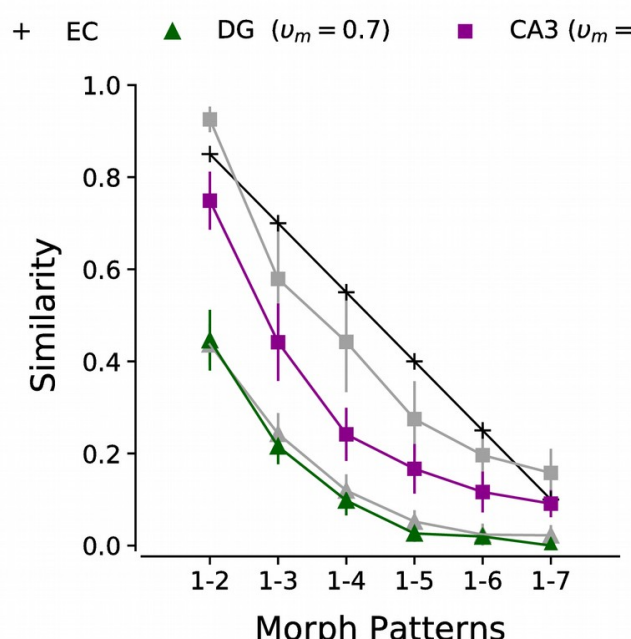

C

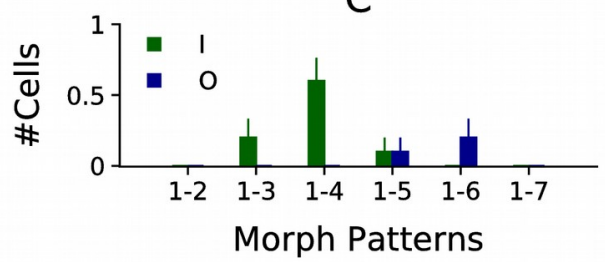

$B$

The S-I-O model

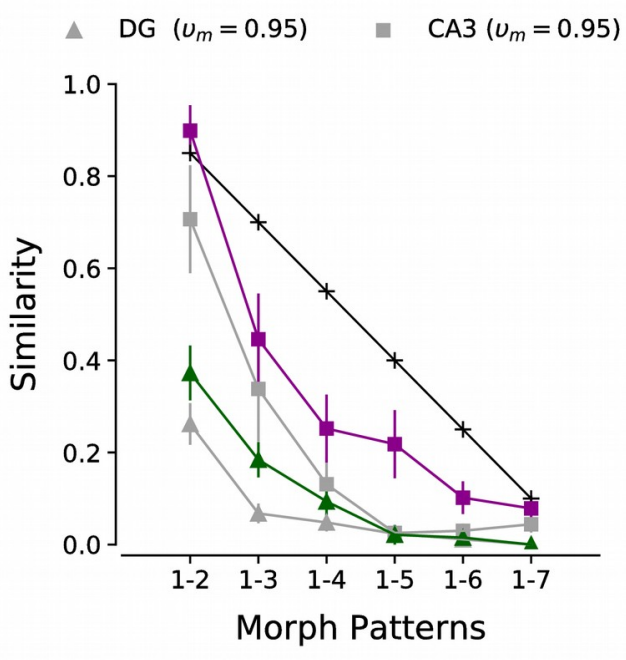

D

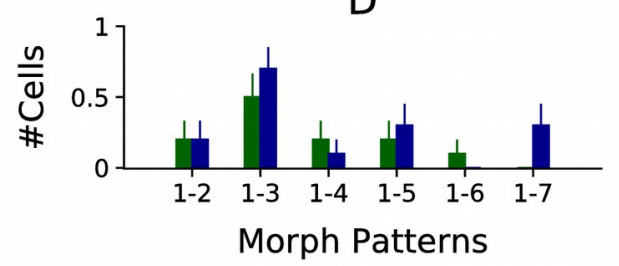

